\title{
Atomic Energy Relations. I*
}

\author{
R. F. BACHER $\dagger$ AND S. GoudSMIT, University of Michigan
}

(Received August 10, 1934)

\begin{abstract}
A simple method for the calculation of approximate energies of atomic levels is presented in this paper. It is based on the derivation of linear relations which express the unknown energy in terms of observed energy values of the atom and its ions. It is shown that the degree of approximation increases with the amount of experimental data available for use in the calculation and also how the best
\end{abstract}

formulas can be obtained for each case. Several tables are given containing formulas for configurations involving $s$ and $p$ electrons. They are applied to the spectra of carbon, nitrogen and oxygen and the energy values so determined are compared with those known from observations. In an appendix the method of approximation is compared with the quantum mechanical perturbation method.

\section{INTRODUCTION}

$\mathrm{T}$ $\mathrm{HE}$ energy states of a system consisting of several electrons in a central field can be found approximately by the use of the perturbation method in the solution of the wave equation. The problem of determining the energy states with an accuracy approaching that with which they are known experimentally is sufficiently complicated of solution, that at present it has been carried out for the two electron case only. For many electrons the problem has been solved in the first order by Slater ${ }^{1}$ and calculations have been made for several elements with wave functions determined by the method of Hartree. These calculations, while remarkably good when the complexity of the problem is considered are still not accurate enough in general to be of much use in the prediction and the correlation of atomic energy states. For this purpose it is desirable to have an easier and more accurate way of obtaining the energy states.

In this article we shall develop approximate relations between the energy states of an atom and its ions. An unknown energy level can then be calculated by expressing it in terms of observed energy values. This is much less difficult than the direct quantum mechanical calculation because the relations derived here are simple linear expressions. Furthermore, the relations possess the favorable property that the

\footnotetext{
* This is the first of a series of articles on this subject. Later papers will contain relations in isoelectronic spectra formulas for configurations involving other than $s$ and $p$ electrons, a special treatment of the cases in which electrons are "lacking" from a complete shell and other extensions of the method presented here.

$\dagger$ At present at Columbia University.

1 J. C. Slater, Phys. Rev. 34, 1293 (1929).
}

degree of accuracy increases rapidly with the amount of experimental information used. In almost all important cases the results are sufficiently accurate to be of value for spectroscopic purposes whereas such accuracy can hardly ever be reached with other methods used at present for the calculation of energies.

The method by which observed energies are used for the prediction of unknown levels is based on principles which are also very simple. In a one-electron atom or ion (that is one electron outside of a core of closed shells), the observed energy levels give direct information about the binding energy of that outer electron. If we add an electron the observed energies of the two-electron ion compared with those of the one-electron ion give information about the change in energy due to the interaction between the two electrons. From the observed energies in three- and more-electron spectra further details about the interaction can be obtained and it is this information which is used for the calculation of unknown energies.

\section{The Many Particle Problem}

(a) Let us consider the one-particle problem, for example one electron outside a core of closed shells. We shall denote its quantum states by $A, B, C$, etc., and the energies to remove the particle by $W(A), W(B), W(C)$, etc. We add a particle and consider next the configuration $A B$ of the two particle system. ${ }^{2}$ If there were no

${ }^{2}$ For the present it is assumed that all degeneracies have been removed. It should further be noted that we assume that it is possible to ascribe each energy level to a definite configuration. This condition is fulfilled in all cases in which this method of approximation can be successfully applied. 
interaction between the two particles the total energy to remove both would be

$$
W_{0}(A B)=W(A)+W(B) .
$$

The energy actually observed, $W(A B)$, differs from this by an amount $w(A B)$ which we shall call "pair energy" and which is defined by the relation

$$
W(A B)=W(A)+W(B)+w(A B) .
$$

The "pair energy" represents the influence of the interaction of the two particles on the total energy.

Let us consider three particles in the configuration $A B C$. If there were no interaction the total energy would be

$$
W_{0}(A B C)=W(A)+W(B)+W(C) .
$$

This will of course be a very rough approximation to the energy actually observed, $W(A B C)$. By using the values of the "pair energies" one is led to a next approximation

$$
\begin{aligned}
W_{1}(A B C)=W(A)+ & W(B)+W(C) \\
& +w(A B)+w(A C)+w(B C)
\end{aligned}
$$$$
\text { or }
$$

$$
\begin{aligned}
W_{1}(A B C)=W(A B) & +W(A C)+W(B C) \\
& -W(A)-W(B)-W(C) .
\end{aligned}
$$

The notation will be simplified by writing $\sum W(\alpha \beta)$ to indicate the sum of two-particle energies and $\sum W(\alpha)$ for the sum of one-particle energies, the sums to be extended over the states of the configuration such as in Eq. (4). In this notation Eq. (4) can be written

$$
W_{1}(A B C)=\sum W(\alpha \beta)-\sum W(\alpha) .
$$

The right sides of Eqs. (4) of (5) contain only observed energies. This expression is often a good approximation to the actual energy $W(A B C)$. The difference between the actual energy and this approximation we shall call the "triple energy," $w(A B C)$. It is defined by the equation

$$
\begin{aligned}
W(A B C)=W(A)+ & W(B)+W(C)+w(A B) \\
& +w(A C)+w(B C)+w(A B C)
\end{aligned}
$$$$
\text { or }
$$

$$
W(A B C)=\sum W(\alpha \beta)-\sum W(\alpha)+w(A B C) .
$$

The "triple energy" represents the change in the total energy due to the influence of the third particle on the interaction of the other two.

In the system of four particles we can set up three successive approximations $W_{0}(A B C D)$, $W_{1}(A B C D)$, and $W_{2}(A B C D)$ which we shall call "zeroth," "first," and "second approximation," respectively. $W_{0}$ uses only the one-electron energies, $W_{1}$ includes the "pair energies" and $W_{2}$ the "pair" and "triple energies." The expressions are

$$
\begin{aligned}
W_{0}(A B C D) & =\sum W(\alpha), \\
W_{1}(A B C D) & =\sum W(\alpha)+\sum w(\alpha \beta) \\
& =\sum W(\alpha \beta)-2 \sum W(\alpha), \\
W_{2}(A B C D) & =\sum W(\alpha)+\sum w(\alpha \beta)+\sum w(\alpha \beta \gamma) \\
& =\sum W(\alpha \beta \gamma)-\sum W(\alpha \beta)+\sum W(\alpha) .
\end{aligned}
$$

The difference between the last approximation and the energy actually observed gives the "quadruple energy," defined by

$$
\begin{aligned}
& W(A B C D)=\sum W(\alpha)+\sum w(\alpha \beta) \\
& +\sum w(\alpha \beta \gamma)+w(A B C D) .
\end{aligned}
$$

The energy of a system containing five particles can be built up in much the same way as those of systems containing fewer particles. $W_{3}(A B C D E)$ appearing in this case for the first time contains in addition to the energy of the pairs and the triples also the energy of the quadruples.

$$
\begin{aligned}
W_{0}(A B C D E) & =\sum W(\alpha), \\
W_{1}(A B C D E) & =\sum W(\alpha \beta)-3 \sum W(\alpha), \\
W_{2}(A B C D E) & =\sum W(\alpha \beta \gamma)-2 \sum W(\alpha \beta) \\
& +3 \sum W(\alpha), \\
W_{3}(A B C D E)=\sum W(\alpha \beta \gamma \delta)-\sum W(\alpha \beta \gamma) & +\sum W(\alpha \beta)-\sum W(\alpha) .
\end{aligned}
$$

It is clear that the procedure which has been followed above can be continued and thus extended to any desired number of particles. Indeed the approximate energy including the interaction in groups one less than the total number of particles may easily be written for the case of $n$ particles. 


$$
\begin{gathered}
W_{n-2}\left(A_{1} A_{2} \cdots A_{n}\right)=\sum W\left(\alpha_{1} \alpha_{2} \cdots \alpha_{n-1}\right) \\
-\sum W\left(\alpha_{1} \alpha_{2} \cdots \alpha_{n-2}\right)+\cdots \\
+(-1)^{n-1} \sum W\left(\alpha_{1} \alpha_{2}\right)+(-1)^{n} \sum W\left(\alpha_{1}\right) .
\end{gathered}
$$

The fact that in all important cases the "pair," "triple," "quadruple energies," etc., form a rapidly decreasing series is the reason for the usefulness of this method of approximation.

It has been tacitly assumed in the relations which have been developed above that it is possible to determine on which states of the $(f-1),(f-2)$, etc., particle systems, a particular state of the $f$ particle system is built. It is necessary to know for each state into what state of the single particle problem it will be transformed if the interaction between the particles is removed. Although this is always uniquely determined theoretically, there are cases where it is not known with certainty. For atoms, this happens when levels of different configurations lie so near together that the large perturbations make it difficult to assign configurations to the individual levels. For the important low energy states of atoms, this difficulty occurs infrequently.

A further difficulty which appears in the atomic problem when only the electrostatic interaction is considered, is the high degree of degeneracy. Due to this degeneracy, it seems at first impossible to determine for a multiplet of a configuration of equivalent electrons, on which multiplet of the ion it is built. This is purely a formal difficulty which can be overcome and it will be discussed later (section III) when the atomic problem is presented in greater detail.

(b) It has been shown in the preceding section how the energy states of the many particle problem can be related to those of the cases of successively less particles down to and including the one particle problem. It is frequently the case, however, that some of the energies which are needed for the determination are not known. In such a case it will be shown that the energy of the given many-particle problem can still be found (somewhat less accurately, to be sure) in terms of those which are known. Suppose that one wishes to express the energy of a state of the five particle problem in terms of those of four, three and two particles, without using the energies of the one particle problem. For this purpose we consider Eq. (9). In the five particle case there are four other equations similar to (9) which may be written for $W_{2}(A B C E), W_{2}(A B D E), W_{2}(A C D E)$ and $W_{2}(B C D E)$. With these five relations it is possible to eliminate the quantities $W(A), W(B)$, $W(C), W(D)$, and $W(E)$ from relation (14). In the result it is necessary to replace the approximate energy $W_{2}(A B C D)$ by the exact energy $W(A B C D)$, and similarly for the others, because the approximate energies involve a knowledge of the single particle energies. The result is given in (16), the energy of the five particle problem now being written $W_{2}^{\prime}(A B C D E)$ due to the approximations that have been made.

$$
\begin{aligned}
& W_{2}{ }^{\prime}(A B C D E)=3 / 4 \sum W(\alpha \beta \gamma \delta) \\
&-1 / 2 \sum W(\alpha \beta \gamma)+1 / 4 \sum W(\alpha \beta) .
\end{aligned}
$$

It is not necessary to go through this lengthy algebraic elimination to obtain (16). Since there are three coefficients which may be adjusted, we can fix them such that the 0th, 1st and 2nd approximations of both sides are identical. The number of approximations which can be balanced in this way depends entirely upon the number of coefficients on the right side of the equation and that may change according to the specific case considered. This observation leads to the following practical method of determining the coefficients. Let us first rewrite (16) with unknown coefficients as in (17).

$$
\begin{aligned}
W_{2}^{\prime}(A B C D E)= & x \sum W(\alpha \beta \gamma \delta) \\
& -y \sum W(\alpha \beta \gamma)+z \sum W(\alpha \beta) .
\end{aligned}
$$

If the 0th order approximations are to be the same on both sides of (17) then the coefficients must be so adjusted that a particular state, say $A$, occurs the same number of times on each side. In the first sum there are four terms containing $A$, in the second sum six and in the last four, and $A$ occurs once on the left side. This gives the numbers needed for (18).

$$
\text { (A) } 1=4 x-6 y+4 z \text {. }
$$

Proceeding similarly with a pair of states, for example $(A B)$ and next with a group of three, $(A B C)$, we get two more equations. 


$$
\begin{array}{ll}
(A B) & 1=3 x-3 y+z \\
(A B C) & 1=2 x-y
\end{array}
$$

The solution of Eqs. (18), (19) and (20) gives the coefficients of (16).

The same method used above to determine the coefficients can be used to get an idea of the relative approximation of the formulas. Let us compare (13) and (16) which give $W_{2}(A B C D E)$ and $W_{2}^{\prime}(A B C D E)$.

$$
\begin{aligned}
& W_{2}(A B C D E)= \sum W(\alpha \beta \gamma)-2 \sum W(\alpha \beta) \\
&+3 \sum W(\alpha), \\
& W_{2}(A B C D E)=3 / 4 \sum W(\alpha \beta \gamma \delta) \\
&-1 / 2 \sum W(\alpha \beta \gamma)+1 / 4 \sum W(\alpha \beta) .
\end{aligned}
$$

Each of these relations is a second approximation, that is, the interaction in groups of three as well as the interaction in pairs has been taken into account. $W_{2}^{\prime}(A B C D E)$, however, is a somewhat better approximation. If we consider the interaction in groups of four, for example in a particular group of four $(A B C D)$, it is seen that the interaction of this group of four occurs once in $W(A B C D E)$. This interaction does not appear at all in the expression given above for $W_{2}(A B C D E)$ while it occurs with a factor $3 / 4$ in the expression for $W_{2}^{\prime}(A B C D E)$, so the latter is a better approximation to the exact energy $W(A B C D E)$ than $W_{2}(A B C D E)$.

In the practical application of these relations to atoms, one seldom knows the energies of all ions down to the single electron case. We shall now show that this method can be used when the energies are only those of the electrons outside the core. Let us take a group of three electrons in states $A, B$ and $C$ outside a core of two electrons in states $D$ and $E$. The symbol * will be used to indicate an energy relative to the core, thus $W^{*}(A B C)$ is defined by (21).

$$
W^{*}(A B C)=W(A B C D E)-W(D E) .
$$

From (4) we can write a relation for $W_{1}^{*}(A B C)$.

$$
\begin{aligned}
W_{1}^{*}(A B C)= & W^{*}(A B)+W^{*}(A C)+W^{*}(B C) \\
& -W^{*}(A)-W^{*}(B)-W^{*}(C) .
\end{aligned}
$$

In terms of total energies including the core (22) may be written as (23).

$$
\begin{array}{r}
W_{1}^{\prime \prime}(A B C D E)=W(A B D E)+W(A C D E) \\
+W(B C D E)-W(A D E)-W(B D E) \\
-W(C D E)+W(D E)
\end{array}
$$

Since the energies of the singles and pairs cancel in this equation, it is a first approximation. In addition, all those groups of three which involve the core states $D$ and $E$ also cancel and the only one which is not considered is the triple $w(A B C)$. This triple energy is just the difference between the exact energy and the first approximation in the three electron problem, so it appears that, including terms of the second approximation, Eq. (22) which expresses the energies of the three electrons relative to the core, neglects the same interaction which is neglected in the problem of three electrons without a core. There are, however, in the problem including the core, several quadruple energies (those not containing both $D$ and $E$ ) and of course the quintuple energy which are also neglected. Since these are in general considerably smaller than the triple energy which was neglected it is apparent that the energies may well be considered relative to the core, and the resulting equation may be expected to hold nearly as well as the similar equation for the same particles without core.

(c) In order to get an idea of the accuracy to be expected from the relations presented above, the method of approximations will be compared with the usual perturbation theory. Table I

TABLE I. Relation of the energy corrections in the recurrence method to those in the perturbation method.

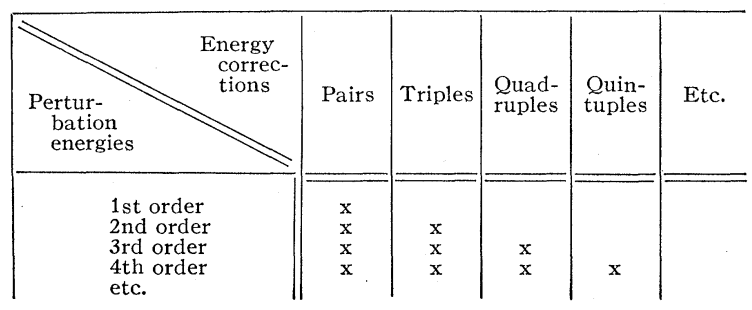

shows the relation between the two methods. The columns in this table give the energy corrections as they have been applied in this paper, first the pair energy, next the triples, etc. The rows list successively the energy corrections given by the ordinary perturbation theory. The energy correction which arises from the source 
indicated at the head of a row or column is split up into all the individual parts, designated by crosses in that row or column. Thus, there is a contribution to the total pair energy from each order of the perturbation starting with the first. It is, indeed, immediately apparent that if the relation considered is a first approximation or better and if the exact energies of the two particle problem are used in that relation, then all the higher orders of the pair interaction have been included. It appears from Table I that the triple energy enters for the first time in the second order of the perturbation theory. It also appears that if the pair energy is considered then the complete first order of the perturbation theory is included. Similarly if the triple energy is considered then the complete second order of the perturbation theory is included, etc. The proof of these statements will be found in Appendix I. It will appear that the first approximation is expected to hold better than a first order calculation by the perturbation theory since the part of the second order which is included in the former is considerable.

\section{IIIa. Absolute Atomic Energies}

The recurrence method described in the preceding section will next be applied to the problem of atomic energy states. The present treatment is limited to those states involving $s$ and $p$ electrons; the states involving $d$ electrons will be treated separately, later. It is essential for the application of the recurrence method to be able to correlate states of successive stages of ionization. The great degeneracy of the problem offers a serious complication. To overcome this complication it is necessary to introduce quantum numbers which describe the system with all degeneracy removed, and second to correlate these non-degenerate states with the degenerate states which are found from experiment. This involves no fundamental difficulties and can be carried out by a procedure which has already been used several times for atomic energy problems and which originated with the wellknown sum relations of Pauli.

Let us consider the problem of an atom with electrostatic and spin-orbit interaction of the electrons neglected. If a magnetic field is intro- duced the degeneracy is completely removed and each electron is in a particular quantum state which is characterized by the quantum numbers $n, l, s, m_{s}$, and $m_{l}$ representing for each electron, respectively, the total quantum number, the orbital quantum number, the spin quantum number and the projections of the spin and orbital momenta on the magnetic field. If we now introduce the electrostatic interaction between the electrons but still neglect the spinorbit interaction (Russell-Saunders coupling), each state is characterized by a particular value of the resultant spin $S$, a particular value of the resultant orbital moment $L$ and their magnetic projection $M_{S}$ and $M_{L}$. It is necessary to find how the states characterized by the quantum numbers $n, l, s, m_{s}$ and $m_{l}$ of the individual electrons are related with those denoted by $S$, $L, M_{S}$ and $M_{L}$.

Since $M_{S}$ and $M_{L}$ are the total projections of the spin and orbital momenta, we have the relations

$$
M_{S}=\sum m_{s}, \quad M_{L}=\sum m_{l} .
$$

The sums are taken over all the electrons. In a few cases where there is only one level with a given $M_{S}$ and $M_{L}$, the sum relations (24) are sufficient to correlate these with the individual electron quantum states. In general, however, this correlation is somewhat more involved.

\section{Two electrons}

Let us first consider two equivalent $p$ electrons. In Table II are gathered all the states of $p^{2}$ for which $M_{S}+M_{L} \geqq 0$. It is known that this configuration gives rise to multiplets ${ }^{3} P(S=1$, $L=1),{ }^{1} D(S=0, L=2)$ and ${ }^{1} S(S=0, L=0)$. The first two columns give the various states of the individual electrons omitting the quantum numbers $n, l$ and $s$. If one of these states has energy $W(A)$ and $W(B)$ for the individual electrons then the particular combination of multiplets found in the last column has the energy $W(A B)$ as is indicated at the bottom of the table. The third column gives the total projections $M_{S}$ and $M_{L}$. The last column gives the multiplet or combination of multiplets which can be assigned to each individual non-degenerate state. It is clear that each of the first four rows are states belonging to ${ }^{3} \mathrm{P}$ since only for ${ }^{3} \mathrm{P}$ is 
TABLE II. Configuration $p^{2}$.

\begin{tabular}{|c|c|c|c|c|c|c|}
\hline$m_{s}{ }^{p}$ & $m_{l}$ & $m_{s}{ }^{p}$ & $m_{l}$ & $M_{S}$ & $M_{L}$ & $\begin{array}{c}p^{2} \\
\text { Multiplets }\end{array}$ \\
\hline $1 / 2$ & 1 & $1 / 2$ & 0 & 1 & 1 & ${ }^{3} P$ \\
\hline $1 / 2$ & 1 & $1 / 2$ & -1 & 1 & 0 & $3 P$ \\
\hline $1 / 2$ & 0 & $1 / 2$ & -1 & 1 & -1 & ${ }^{3} P$ \\
\hline$-1 / 2$ & 1 & $-1 / 2$ & 0 & -1 & 1 & ${ }^{3} P$ \\
\hline $1 / 2$ & 1 & $-1 / 2$ & 1 & 0 & 2 & ${ }^{1} D$ \\
\hline $\begin{array}{l}1 / 2 \\
1 / 2\end{array}$ & $\begin{array}{l}1 \\
0\end{array}$ & $\begin{array}{l}-1 / 2 \\
-1 / 2\end{array}$ & $\begin{array}{l}0 \\
1\end{array}$ & 0 & 1 & $\begin{array}{l}1 / 2{ }^{3} P+1 / 2{ }^{1} D \\
1 / 2{ }^{3} P+1 / 2{ }^{1} D\end{array}$ \\
\hline $\begin{array}{l}1 / 2 \\
1 / 2 \\
1 / 2\end{array}$ & $\begin{array}{r}1 \\
0 \\
-1\end{array}$ & $\begin{array}{l}-1 / 2 \\
-1 / 2 \\
-1 / 2\end{array}$ & $\begin{array}{r}-1 \\
0 \\
1\end{array}$ & 0 & 0 & $\begin{array}{r}1 / 2{ }^{3} P+1 / 6{ }^{1} D+1 / 3 \\
2 / 3 \\
1 D+1 / 3 \\
1 / 2 \\
1 / 2 P+1 / 6{ }^{3} D+1 / 3\end{array}$ \\
\hline \multicolumn{2}{|c|}{$W(A)$} & \multicolumn{2}{|c|}{$W(B)$} & $M_{S}$ & $M_{L}$ & $W(A B)$ \\
\hline
\end{tabular}

there a projection $M_{S}= \pm 1$. Similarly the fifth state belongs to ${ }^{1} D$ since only for ${ }^{1} D$ is there a projection $M_{L}=2$. The assignment of the combination of multiplets to the states with $M_{S}=0$, $M_{L}=1$ and those with $M_{S}=0, M_{L}=0$ cannot be made in this way. To the first set belong ${ }^{3} P$ and ${ }^{1} D$ and to the second ${ }^{3} P,{ }^{1} D$ and ${ }^{1} S$. The coefficients in the linear combinations which are correlated with each state can be found by standard quantum mechanical methods, for example by the famous $\xi, \eta$ method. They can also be found directly from the recurrence method, as is demonstrated in Appendix 2. Since we neglect the spin-orbit interaction, it is not necessary to distinguish between the various $J$-levels of each multiplet. In order to find the energies it is not necessary to know the coefficients for the $M_{S}=0, M_{L}=1$ group since ${ }^{3} P$ and ${ }^{1} D$ are determined elsewhere, but for the determination of the coefficients in more complicated configurations directly from the recurrence method, it is necessary to know the proper linear combination for each individual state. It should be noted that the sum of the coefficients for each state, as well as the sum of the coefficients of a particular multiplet in a group with given $M_{S}$ and $M_{L}$, is 1 .

Table III gives the configuration $s p$, showing the linear combination of ${ }^{3} P$ and ${ }^{1} P$ for each non-degenerate state with $M_{S}+M_{L} \geqq 0$. All those states with $M_{S}= \pm 1$ are ${ }^{3} P$ alone, while the two sets with $M_{S}=0$ are ${ }^{3} P$ and ${ }^{1} P$ together. The coefficients in the linear combinations can be found in a way similar to that used above for $p^{2}$ or they can be written directly as $1 / 2$, since one state with $M_{S}=0$ and $M_{L}=1$ can be ob-
TABLE III. Configuration $s p$.

\begin{tabular}{|c|c|c|c|c|c|}
\hline$\stackrel{s}{m_{s}}$ & $m_{s}^{p}$ & $m_{l}$ & $M_{S}$ & $M_{L}$ & $\stackrel{s p}{\text { Multiplets }}$ \\
\hline $1 / 2$ & $1 / 2$ & 1 & 1 & 1 & ${ }^{3} P$ \\
\hline $1 / 2$ & $1 / 2$ & 0 & 1 & 0 & ${ }^{3} P$ \\
\hline $1 / 2$ & $1 / 2 \quad-$ & -1 & 1 & -1 & ${ }^{3} P$ \\
\hline$-1 / 2$ & $-1 / 2$ & 1 & -1 & 1 & ${ }^{3} P$ \\
\hline $\begin{array}{r}1 / 2 \\
-1 / 2\end{array}$ & $\begin{array}{r}-1 / 2 \\
1 / 2\end{array}$ & $\begin{array}{l}1 \\
1\end{array}$ & 0 & 1 & $\begin{array}{l}1 / 2{ }^{3} P+1 / 2{ }^{1} P \\
1 / 2{ }^{3} P+1 / 2{ }^{1} P\end{array}$ \\
\hline $\begin{array}{r}1 / 2 \\
-1 / 2\end{array}$ & $\begin{array}{r}-1 / 2 \\
1 / 2\end{array}$ & $\begin{array}{l}0 \\
0\end{array}$ & 0 & 0 & $\begin{array}{l}1 / 2{ }^{3} P+1 / 2{ }^{1} P \\
1 / 2{ }^{3} P+1 / 2{ }^{1} P\end{array}$ \\
\hline$W(A)$ & $W(B)$ & & $M_{S}$ & $M_{L}$ & $W(A B)$ \\
\hline
\end{tabular}

tained from the other by changing the signs of the spins. The same is true for the two states with $M_{S}=0$ and $M_{L}=0$. It is not necessary to give a table for $s^{2}$ since this gives but one multiplet, ${ }^{1} S$. With the help of these tables for the two electron systems, we can now build configurations of more than two electrons, restricting ourselves at present to $s$ electrons and to equivalent $p$ electrons.

\section{Three electrons}

The first three electron system to be considered is the configuration $p^{3}$ which gives the multiplets ${ }^{4} S,{ }^{2} D$ and ${ }^{2} P$. The first three columns of Table IV give the individual electron states $A, B$ and $C$. The fifth column gives the linear combination of multiplets assigned to each state. The first state is uniquely ${ }^{4} S$ and the second ${ }^{2} D$. The assignment of linear combinations to the other states is discussed in detail in Appendix 2. The last column of Table IV gives the sum of the two electron states $(A B)+(A C)+(B C)$ on which the three electron states are built. For example, if we consider the first state we find that each of the three possible two electron states belong to $p^{2}{ }^{3} P$ in Table II.

The configuration $s p^{2}$ gives the multiplets ${ }^{4} P$, ${ }^{2} P,{ }^{2} D$ and ${ }^{2} S$. The first three columns of Table $\mathrm{V}$ give the states of the individual electrons. The fifth column gives the linear combination of multiplets corresponding to each state. Each of the first three states is uniquely ${ }^{4} P$ and each of the next two is uniquely ${ }^{2} D$. The proper linear combination to be assigned to the other states is discussed in Appendix 2. The last two columns give the multiplets of $p^{2}$ and $s p$, respectively, on which the three electron states of $s p^{2}$ are built.

Table VI gives the non-degenerate states of 
TABLE IV. Configuration $p^{3}$.

\begin{tabular}{|c|c|c|c|c|c|c|c|c|c|}
\hline$m_{s}$ & $m_{l}$ & $m_{s}{ }^{p}$ & $m_{l}$ & $m_{s}{ }^{p}$ & $m_{l}$ & $M_{S}$ & $M_{L}$ & $\begin{array}{c}p^{3} \\
\text { Multiplets }\end{array}$ & Multiplets from pairs \\
\hline $1 / 2$ & 1 & $1 / 2$ & 0 & $1 / 2$ & -1 & $3 / 2$ & 0 & ${ }^{4} S$ & $3{ }^{3} P$ \\
\hline $1 / 2$ & 1 & $1 / 2$ & 0 & $-1 / 2$ & 1 & $1 / 2$ & 2 & ${ }^{2} D$ & $3 / 2{ }^{3} P+3 / 2{ }^{1} D$ \\
\hline $\begin{array}{l}1 / 2 \\
1 / 2\end{array}$ & $\begin{array}{l}1 \\
1\end{array}$ & $\begin{array}{l}1 / 2 \\
1 / 2\end{array}$ & $\begin{array}{r}0 \\
-1\end{array}$ & $\begin{array}{l}-1 / 2 \\
-1 / 2\end{array}$ & $\begin{array}{l}0 \\
1\end{array}$ & $1 / 2$ & 1 & $\begin{array}{l}1 / 22 D+1 / 22 P \\
1 / 22 D+1 / 22 P\end{array}$ & $\begin{array}{l}3 / 2{ }^{3} P+7 / 6^{1} D+1 / 3{ }^{1} S \\
3 / 2{ }^{3} P+7 / 6^{1} D+1 / 3{ }^{1} S\end{array}$ \\
\hline $1 / 2$ & 1 & $-1 / 2$ & 1 & $-1 / 2$ & 0 & $-1 / 2$ & 2 & ${ }^{2} D$ & $3 / 2{ }^{3} P+3 / 2^{1} D$ \\
\hline $\begin{array}{l}1 / 2 \\
1 / 2\end{array}$ & $\begin{array}{l}1 \\
0\end{array}$ & $\begin{array}{l}-1 / 2 \\
-1 / 2\end{array}$ & $\begin{array}{l}1 \\
1\end{array}$ & $\begin{array}{l}-1 / 2 \\
-1 / 2\end{array}$ & $\begin{array}{r}-1 \\
0\end{array}$ & $-1 / 2$ & 1 & $\begin{array}{l}1 / 2{ }^{2} D+1 / 2{ }^{2} P \\
1 / 2{ }^{2} D+1 / 2{ }^{2} P\end{array}$ & 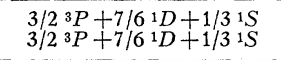 \\
\hline $\begin{array}{l}1 / 2 \\
1 / 2 \\
1 / 2\end{array}$ & $\begin{array}{l}1 \\
1 \\
0\end{array}$ & $\begin{array}{l}1 / 2 \\
1 / 2 \\
1 / 2\end{array}$ & $\begin{array}{r}0 \\
-1 \\
-1\end{array}$ & $\begin{array}{l}-1 / 2 \\
-1 / 2 \\
-1 / 2\end{array}$ & $\begin{array}{r}-1 \\
0 \\
1\end{array}$ & $1 / 2$ & 0 & $\begin{array}{l}1 / 3{ }^{4} S+1 / 6^{2} D+1 / 2^{2} P \\
1 / 3{ }^{4} S+2 / 3^{2} D \\
1 / 3{ }^{4} S+1 / 6^{2} D+1 / 2^{2} P\end{array}$ & $\begin{array}{l}23^{3} P+2 / 3{ }^{1} D+1 / 31 S \\
2{ }^{3} P+1 D \\
2{ }^{3} P+2 / 3{ }^{1} D+1 / 31 S\end{array}$ \\
\hline \multicolumn{2}{|c|}{$W(A)$} & \multicolumn{2}{|c|}{$W(B)$} & \multicolumn{2}{|c|}{$W(C)$} & $M_{S}$ & $M_{L}$ & $W(A B C)$ & $W(A B)+W(A C)+W(B C)$ \\
\hline
\end{tabular}

TABLE V. Configuration $s p^{2}$.

\begin{tabular}{|c|c|c|c|c|c|c|c|c|c|}
\hline \multirow{2}{*}{$\begin{array}{l}s \\
m_{s} \\
1 / 2\end{array}$} & \multirow{2}{*}{$\frac{m_{s}}{1 / 2}$} & \multirow{2}{*}{$\frac{m_{l}}{1}$} & $m_{s}{ }^{p}$ & $m_{l}$ & \multirow{2}{*}{$\frac{M_{S}}{3 / 2}$} & \multirow{2}{*}{$\frac{M_{L}}{1}$} & $\begin{array}{c}s p^{2} \\
\text { Multiplets }\end{array}$ & \multicolumn{2}{|c|}{$p^{2}$ Multiplets from pairs } \\
\hline & & & $1 / 2$ & 0 & & & ${ }^{4} P$ & ${ }^{3} P$ & $2{ }^{3} P$ \\
\hline $1 / 2$ & $1 / 2$ & 1 & $1 / 2$ & -1 & $3 / 2$ & 0 & ${ }^{4 P}$ & ${ }^{3} P$ & $2{ }^{3} P$ \\
\hline $1 / 2$ & $1 / 2$ & 0 & $1 / 2$ & -1 & $3 / 2$ & -1 & ${ }^{4} P$ & ${ }^{3} P$ & $2{ }^{3} P$ \\
\hline $1 / 2$ & $1 / 2$ & 1 & $-1 / 2$ & 1 & $1 / 2$ & 2 & ${ }^{2} D$ & ${ }^{1} D$ & $3 / 2^{3} P+1 / 2{ }^{1} P$ \\
\hline$-1 / 2$ & $1 / 2$ & 1 & $-1 / 2$ & 1 & $-1 / 2$ & 2 & ${ }^{2} D$ & ${ }^{1} D$ & $3 / 2^{3} P+1 / 2{ }^{1} P$ \\
\hline $\begin{array}{r}1 / 2 \\
1 / 2 \\
-1 / 2\end{array}$ & $\begin{array}{l}1 / 2 \\
1 / 2 \\
1 / 2\end{array}$ & $\begin{array}{l}1 \\
0 \\
1\end{array}$ & $\begin{array}{r}-1 / 2 \\
-1 / 2 \\
1 / 2\end{array}$ & $\begin{array}{l}0 \\
1 \\
0\end{array}$ & $1 / 2$ & 1 & $\begin{array}{r}1 / 3{ }^{4} P+1 / 2{ }^{2} D+1 / 6{ }^{2} P \\
1 / 3{ }^{4} P+1 / 2{ }^{2} D+1 / 62 P \\
1 / 3{ }^{4} P \\
+2 / 3{ }^{2} P\end{array}$ & $\begin{array}{l}1 / 2{ }^{3 P}+1 / 21 D \\
1 / 2{ }_{3 P} P+1 / 2{ }^{1} D \\
\quad{ }^{2} P\end{array}$ & $\begin{array}{c}3 / 2{ }^{3} P+1 / 2{ }^{1 P} \\
3 / 2{ }^{3} P+1 / 21 P \\
3 P+\quad 1 P \\
3 P+19\end{array}$ \\
\hline $\begin{array}{r}1 / 2 \\
1 / 2 \\
1 / 2 \\
-1 / 2\end{array}$ & $\begin{array}{l}1 / 2 \\
1 / 2 \\
1 / 2 \\
1 / 2\end{array}$ & $\begin{array}{r}1 \\
0 \\
-1 \\
1\end{array}$ & $\begin{array}{r}-1 / 2 \\
-1 / 2 \\
-1 / 2 \\
1 / 2\end{array}$ & $\begin{array}{r}-1 \\
0 \\
1 \\
-1\end{array}$ & $1 / 2$ & 0 & $\begin{array}{l}1 / 3{ }^{4} P+1 / 6^{2} D+1 / 6^{2} P+1 / 3{ }^{2} S \\
2 / 3{ }^{2} D+1 / 3{ }^{2} S \\
1 / 3{ }^{4} P+1 / 6^{2} D+1 / 6{ }^{2} P+1 / 3{ }^{2} S \\
1 / 3{ }^{4} P+2 / 3{ }^{2} P\end{array}$ & $\begin{array}{l}1 / 2{ }^{3} P+1 / 6{ }^{1} D+1 / 3{ }^{1 S} \\
2 / 3{ }^{1} D+1 / 3 \\
1 / 2 S \\
{ }^{3} P+1 / 6{ }^{1} D+1 / 3{ }^{1} S \\
{ }^{3} P\end{array}$ & $\begin{array}{l}3 / 2{ }^{3 P} P+1 / 2{ }^{1} P \\
3 / 2{ }^{3 P}+1 / 2 \cdot{ }^{1} \\
3 / 2{ }^{3} P+1 / 2 \cdot{ }^{3} P \\
{ }^{3} P+\quad{ }^{1} P\end{array}$ \\
\hline$W(A)$ & & B) & $W$ & & $M S$ & $M_{L}$ & $W(A B C)$ & $W(B C)$ & $W(A B)+W(A C)$ \\
\hline
\end{tabular}

$s^{2} p$ and is very simple since the two $s$ electrons form a closed shell.

With the information available in Tables IV, $\mathrm{V}$ and VI it is now possible to write the energies of the three electron problem directly from Eq. (4). From the first state of Table IV, for example, we may write

$$
W_{1}\left(p^{3}{ }^{4} S\right)=3 W\left(p^{2}{ }^{3} P\right)-3 W\left(p^{2} P\right) .
$$

It is important to note that these energies must be measured either with respect to the naked nucleus or to the core, if there is one inside the $p$ electrons. From the second state of Table IV we have

TABLE VI. Configuration $s^{2} p$.

\begin{tabular}{|c|c|c|c|c|c|c|c|c|}
\hline \multirow{2}{*}{$\begin{array}{c}s \\
m_{s}\end{array}$} & \multirow{2}{*}{$\begin{array}{c}s \\
m_{s}\end{array}$} & \multicolumn{2}{|c|}{$p$} & \multirow[b]{2}{*}{$M_{S}$} & \multirow[b]{2}{*}{$M_{L}$} & \multirow{2}{*}{$\begin{array}{c}s^{2} p \\
\text { multiplets }\end{array}$} & \multicolumn{2}{|c|}{$\begin{array}{l}\text { Multiplets from } \\
\text { pairs }\end{array}$} \\
\hline & & $m_{s}$ & $m_{l}$ & & & & $s^{2}$ & $s p$ \\
\hline $\begin{array}{l}1 / 2 \\
1 / 2 \\
1 / 2\end{array}$ & $\begin{array}{l}-1 / 2 \\
-1 / 2 \\
-1 / 2\end{array}$ & $\begin{array}{r}1 / 2 \\
1 / 2 \\
-1 / 2\end{array}$ & $\begin{array}{l}1 \\
0 \\
1\end{array}$ & $\begin{array}{r}1 / 2 \\
1 / 2 \\
-1 / 2\end{array}$ & $\begin{array}{l}1 \\
0 \\
1\end{array}$ & $\begin{array}{l}2 P \\
2 P \\
2 P\end{array}$ & $\begin{array}{l}1 S \\
1 S \\
1 S\end{array}$ & 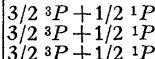 \\
\hline & & & & & & & & \\
\hline
\end{tabular}

$W_{1}\left(p^{3}{ }^{2} D\right)=3 / 2 W\left(p^{2}{ }^{3} P\right)$

$$
+3 / 2 W\left(p^{2} 1 D\right)-3 W\left(p^{2} P\right) .
$$

$W_{i}\left(p^{3} 2\right)$ can easily be found by elimination from the other states which are known in Table IV. The energies of the various multiplets of $p^{3}$, $s p^{2}, s^{2} p$ and $s^{2} s^{\prime}$ found in this way are presented in Table VII. The $W$ 's are omitted in Table VII for convenience.

\begin{tabular}{|c|c|c|c|c|c|}
\hline & \multicolumn{2}{|r|}{$p^{2}$} & $s p$ & $p$ & $s$ \\
\hline $\begin{array}{r}p^{3} 4 S= \\
2 D= \\
2 P=\end{array}$ & \multicolumn{2}{|c|}{ 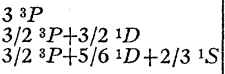 } & & $\begin{array}{l}-32 P \\
-32 P \\
-32 P\end{array}$ & \\
\hline \multirow[t]{2}{*}{$\begin{array}{r}s p^{2}{ }^{4} P= \\
2 P= \\
2 D= \\
2 S=\end{array}$} & $\begin{array}{l}3 P \\
{ }^{3} P\end{array}$ & ${ }^{1} D$ & $\mid \begin{array}{l}2{ }^{3} P \\
1 / 2{ }^{3} P+3 / 21 P \\
3 / 2{ }^{3} P+1 / 21 P \\
3 / 2{ }^{3} P+1 / 2{ }^{1} P\end{array}$ & $\begin{array}{l}-22 P \\
-22 P \\
-22 P \\
-22 P\end{array}$ & $\begin{array}{l}-2 S \\
-2 S \\
-2 S \\
-2 S\end{array}$ \\
\hline & $s^{2}$ & $s s^{\prime}$ & $s p$ & $p$ & $s$ and $s^{\prime}$ \\
\hline $\begin{array}{l}s^{2} p^{2} P= \\
s^{2} s^{\prime}{ }^{2} S=\end{array}$ & $\begin{array}{l}{ }^{1 S} \\
{ }^{S} S\end{array}$ & $3 / 2{ }^{3} S+1 / 2{ }^{1} S$ & $3 / 2{ }^{3} P+1 / 2{ }^{1} P$ & $-2 P$ & $\begin{array}{l}-2{ }^{2} S \\
-2{ }^{2} S-2 S^{\prime}\end{array}$ \\
\hline
\end{tabular}

TABLE VII. Results for three electrons. 
TABLE VIII. Configuration $p^{4}$.

\begin{tabular}{|c|c|c|c|c|c|c|c|}
\hline $\begin{array}{c}\stackrel{p}{p} \\
m_{s}\end{array} m_{l}$ & $\begin{array}{cc}p & \\
m_{s} & m_{l}\end{array}$ & $\underset{m_{s}}{p}$ & $m l$ & $m_{8}^{p}$ & $m_{l}$ & $M_{S} M_{L}$ & Multiplets \\
\hline $1 / 2 \quad 1$ & $1 / 2 \quad 0$ & $1 / 2$ & -1 & $-1 / 2$ & 1 & 1 & ${ }^{3} P$ \\
\hline $1 / 2 \quad 1$ & $1 / 2 \quad 0$ & $1 / 2$ & -1 & $-1 / 2$ & 0 & 0 & ${ }^{3} P$ \\
\hline $1 / 2 \quad 1$ & $1 / 2 \quad 0$ & $1 / 2$ & -1 & $-1 / 2$ & -1 & $\begin{array}{ll}1 & -1\end{array}$ & ${ }^{3} P$ \\
\hline $\begin{array}{ll}-1 / 2 & 1\end{array}$ & $-1 / 2 \quad 0$ & $-1 / 2$ & -1 & $1 / 2$ & -1 & -1 & ${ }^{3} P$ \\
\hline $1 / 2 \quad 1$ & 120 & $-1 / 2$ & 1 & $-1 / 2$ & 0 & 0 & ${ }^{1} D$ \\
\hline $\begin{array}{ll}1 / 2 & 1 \\
1 / 2 & 1\end{array}$ & $\begin{array}{rr}1 / 2 & -1 \\
1 / 2 & 0\end{array}$ & $\begin{array}{l}-1 / 2 \\
-1 / 2\end{array}$ & $\begin{array}{l}1 \\
1\end{array}$ & $\begin{array}{l}-1 / 2 \\
-1 / 2\end{array}$ & $\begin{array}{r}0 \\
-1\end{array}$ & 1 & $\begin{array}{l}1 / 2{ }^{3} P+1 / 2{ }^{1} D \\
1 / 2{ }^{3} P+1 / 2{ }^{1} D\end{array}$ \\
\hline $\begin{array}{ll}1 / 2 & 1 \\
1 / 2 & 1 \\
1 / 2 & 0\end{array}$ & $\begin{array}{rr}1 / 2 & -1 \\
1 / 2 & 0 \\
1 / 2 & -1\end{array}$ & $\begin{array}{l}-1 / 2 \\
-1 / 2 \\
-1 / 2\end{array}$ & $\begin{array}{l}1 \\
0 \\
1\end{array}$ & $\begin{array}{l}-1 / 2 \\
-1 / 2 \\
-1 / 2\end{array}$ & \begin{tabular}{r|}
-1 \\
-1 \\
0
\end{tabular} & 0 & $\begin{array}{c}2 / 3{ }^{1} D+1 / 31 S \\
1 / 2{ }^{3} P+1 / 61 D \\
+1 / 3{ }^{1 S} \\
\text { same }\end{array}$ \\
\hline (A) & (B) & (C) & & (D) & & & $(A B C D)$ \\
\hline
\end{tabular}

\section{Four electrons}

The configuration $p^{4}$ gives rise to the multiplets ${ }^{3} P,{ }^{1} D$ and ${ }^{1} S$. Table VIII shows the various states of the individual electrons and the linear combination of the multiplets which represents each state with $M_{S}+M_{L} \geqq 0$. Table IX is a similar table for the configuration $s p^{3}$. The energies of the various multiplets of $p^{4}, s p^{3}$ and also $s^{2} p^{2}$ in terms of the three, two and one electron energies are gathered in Table $\mathrm{X}$. These relations include the single, pair and triple energies and should therefore be rather good approximations to the actual energies of the states. In the notation of the preceding section these relations are second approximations and should be written, for example, $W_{2}\left(p^{4}{ }^{3} P\right)$.

The relations given in Table $\mathrm{X}$ are suitable for finding the energy of a state of either the four, three, two or one electron system in terms of the others. It may happen that there is not sufficient information and it is desired to get a relation, say, between the four, three and two electron states. Relations which give the relative energies for this case can be obtained directly from Table $\mathrm{X}$, by subtraction. If we wish the absolute energies we can get them in the following way for example for $p^{4} 3$. From Table $\mathrm{X}$ we have

$$
\begin{array}{r}
W_{2}\left(p^{4}{ }^{3} P\right)=4 / 3\left(p^{3}{ }^{4} S\right)+5 / 3\left(p^{3}{ }^{2} D\right) \\
+\left(p^{3}{ }^{2} P\right)-4\left(p^{2}{ }^{3} P\right)-5 / 3\left(p^{2}{ }^{1} D\right) \\
-1 / 3\left(p^{2}{ }^{1} S\right)+4\left(p^{2} P\right)
\end{array}
$$

The energies given here are all relative to the closed shell beneath. If we rewrite Eq. (27) giving the energies relative to the ion $p^{2} P$ we find that the terms $\left(p^{2} P\right)$ cancel with the exception of a single one.

$$
\begin{array}{r}
W_{2}{ }^{*}\left(p^{4}{ }^{3} P\right)=4 / 3\left(p^{3}{ }^{4} S^{*}\right)+5 / 3\left(p^{3}{ }^{2} D^{*}\right) \\
+\left(p^{3}{ }^{2} P^{*}\right)-4\left(p^{2}{ }^{3} P^{*}\right)-5 / 3\left(p^{2}{ }^{1} D^{*}\right) \\
-1 / 3\left(p^{2}{ }^{1} S^{*}\right)+\left(p^{2} P\right)
\end{array}
$$

\begin{tabular}{|c|c|c|c|c|c|c|c|c|c|c|}
\hline $\begin{array}{c}s \\
m_{s}\end{array}$ & $m_{s}^{p}$ & $m_{l}$ & $m_{s}{ }^{p}$ & $m_{l}$ & $m_{s}{ }^{p}$ & $m_{l}$ & $M_{S}$ & $M_{L}$ & \multicolumn{2}{|c|}{$s p^{3}$ Multiplets } \\
\hline $1 / 2$ & $1 / 2$ & 1 & $1 / 2$ & 0 & $1 / 2$ & -1 & 2 & 0 & \multicolumn{2}{|l|}{${ }^{5} S$} \\
\hline $1 / 2$ & $1 / 2$ & 1 & $1 / 2$ & 0 & $-1 / 2$ & 1 & 1 & 2 & \multicolumn{2}{|l|}{${ }^{3} D$} \\
\hline $\begin{array}{r}-1 / 2 \\
1 / 2\end{array}$ & $\begin{array}{l}1 / 2 \\
1 / 2\end{array}$ & $\begin{array}{l}1 \\
1\end{array}$ & $\begin{array}{r}1 / 2 \\
-1 / 2\end{array}$ & $\begin{array}{l}0 \\
0\end{array}$ & $\begin{array}{l}-1 / 2 \\
-1 / 2\end{array}$ & $\begin{array}{l}1 \\
1\end{array}$ & 0 & 2 & \multicolumn{2}{|c|}{$\begin{array}{l}1 / 2{ }^{3} D+1 / 2{ }^{1} D \\
1 / 2{ }^{3} D+1 / 2{ }^{1} D \\
\end{array}$} \\
\hline $\begin{array}{l}1 / 2 \\
1 / 2\end{array}$ & $\begin{array}{l}1 / 2 \\
1 / 2\end{array}$ & $\begin{array}{l}1 \\
1\end{array}$ & $\begin{array}{l}1 / 2 \\
1 / 2\end{array}$ & $\begin{array}{r}0 \\
-1\end{array}$ & $\begin{array}{l}-1 / 2 \\
-1 / 2\end{array}$ & $\begin{array}{l}0 \\
1\end{array}$ & 1 & 1 & $\begin{array}{l}1 / 2{ }^{3} D \\
1 / 2{ }^{3} D\end{array}$ & $\begin{array}{l}+1 / 2{ }^{3} P \\
+1 / 2{ }^{3} P\end{array}$ \\
\hline $\begin{array}{r}1 / 2 \\
1 / 2 \\
1 / 2 \\
-1 / 2\end{array}$ & $\begin{array}{l}1 / 2 \\
1 / 2 \\
1 / 2 \\
1 / 2\end{array}$ & $\begin{array}{l}1 \\
0 \\
1 \\
1\end{array}$ & $\begin{array}{l}1 / 2 \\
1 / 2 \\
1 / 2 \\
1 / 2\end{array}$ & $\begin{array}{r}0 \\
-1 \\
-1 \\
0\end{array}$ & $\begin{array}{r}-1 / 2 \\
-1 / 2 \\
-1 / 2 \\
1 / 2\end{array}$ & $\begin{array}{r}-1 \\
1 \\
0 \\
-1\end{array}$ & 1 & 0 & $\begin{array}{l}1 / 4{ }^{5} S+1 / 12{ }^{3} S+1 / 6{ }^{3} D \\
1 / 4 \\
1 / 4 S+1 / 12{ }^{5} S+2 / 3{ }^{3} D \\
1 / 4{ }^{5} S+3 / 4{ }^{3} S\end{array}$ & $+1 / 2{ }^{3} P$ \\
\hline $\begin{array}{r}-1 / 2 \\
-1 / 2 \\
1 / 2 \\
1 / 2\end{array}$ & $\begin{array}{l}1 / 2 \\
1 / 2 \\
1 / 2 \\
1 / 2 \\
\end{array}$ & $\begin{array}{l}1 \\
1 \\
1 \\
0\end{array}$ & $\begin{array}{r}1 / 2 \\
1 / 2 \\
-1 / 2 \\
-1 / 2 \\
\end{array}$ & $\begin{array}{r}0 \\
-1 \\
1 \\
1 \\
\end{array}$ & $\begin{array}{l}-1 / 2 \\
-1 / 2 \\
-1 / 2 \\
-1 / 2 \\
\end{array}$ & $\begin{array}{r}0 \\
1 \\
-1 \\
0\end{array}$ & 0 & 1 & \multicolumn{2}{|c|}{$\begin{array}{c}1 / 4^{3} D+1 / 4^{1} D+1 / 4^{3} P+1 / 4{ }^{1} P \\
\text { same } \\
\dddot{~}\end{array}$} \\
\hline $\begin{array}{r}-1 / 2 \\
-1 / 2 \\
1 / 2 \\
1 / 2 \\
-1 / 2 \\
1 / 2 \\
\end{array}$ & $\begin{array}{r}1 / 2 \\
1 / 2 \\
-1 / 2 \\
-1 / 2 \\
1 / 2 \\
-1 / 2 \\
\end{array}$ & $\begin{array}{l}1 \\
0 \\
1 \\
0 \\
1 \\
1\end{array}$ & $\begin{array}{r}1 / 2 \\
1 / 2 \\
-1 / 2 \\
-1 / 2 \\
1 / 2 \\
-1 / 2 \\
\end{array}$ & $\begin{array}{r}0 \\
-1 \\
0 \\
-1 \\
-1 \\
-1\end{array}$ & $\begin{array}{r}-1 / 2 \\
-1 / 2 \\
1 / 2 \\
1 / 2 \\
-1 / 2 \\
1 / 2\end{array}$ & $\begin{array}{r}-1 \\
1 \\
-1 \\
1 \\
0 \\
0\end{array}$ & 0 & 0 & \multicolumn{2}{|c|}{$\begin{array}{c}1 / 6{ }^{5} S+1 / 6{ }^{3} S+1 / 12{ }^{3} D+1 / 12{ }^{1} D+1 / 4{ }^{3} P+1 / 4{ }^{1} P \\
\text { same } \\
1 / 6{ }^{5} S+1 / 6{ }^{3} S+1 / 3{ }^{3} D+1 / 3{ }^{1} D \\
\text { same }\end{array}$} \\
\hline $\begin{array}{l}1 / 2 \\
1 / 2\end{array}$ & $\begin{array}{l}1 / 2 \\
1 / 2\end{array}$ & $\begin{array}{l}0 \\
1\end{array}$ & $\begin{array}{l}1 / 2 \\
1 / 2\end{array}$ & $\begin{array}{l}-1 \\
-1\end{array}$ & $\begin{array}{l}-1 / 2 \\
-1 / 2\end{array}$ & $\begin{array}{r}0 \\
-1\end{array}$ & 1 & -1 & $\begin{array}{l}1 / 2{ }^{3} D \\
1 / 2{ }^{3} D \\
\end{array}$ & $\begin{array}{l}+1 / 2{ }^{3} P \\
+1 / 2{ }^{3 P} \\
\end{array}$ \\
\hline$(A)$ & \multicolumn{2}{|l|}{$(B)$} & \multicolumn{2}{|l|}{$(C)$} & \multicolumn{2}{|l|}{$(D)$} & . & & \multicolumn{2}{|c|}{$(A B C D)$} \\
\hline
\end{tabular}

TABLE IX. Configuration $s p^{3}$. 
TABLE X. Results for four electrons.

\begin{tabular}{|c|c|c|c|c|c|c|c|c|c|c|}
\hline & \multicolumn{2}{|c|}{$p^{3}$} & \multicolumn{2}{|l|}{$s p^{2}$} & \multicolumn{2}{|r|}{$p^{2}$} & \multicolumn{2}{|c|}{$s p$} & $p$ & $s$ \\
\hline $\begin{array}{r}p^{4} 3 P= \\
1 D= \\
1 S=\end{array}$ & \multicolumn{2}{|c|}{$\begin{array}{r}4 / 3{ }^{4} S+5 / 3{ }^{2} D+{ }^{2 P} \\
3^{2} D+{ }^{2 P} \\
4{ }^{2} P\end{array}$} & \multicolumn{2}{|l|}{. } & \multicolumn{2}{|c|}{$\begin{array}{l}-4{ }^{3} P-5 / 31 D-1 / 31 S \\
-3{ }^{3} P-8 / 31 D-1 / 31 S \\
-3{ }^{3} P-5 / 31 D-4 / 31 S \\
\end{array}$} & & & $\begin{array}{l}+4^{2} P \\
+42 P \\
+4^{2} P\end{array}$ & . \\
\hline \multirow[t]{2}{*}{$\begin{array}{r}s p^{3} S= \\
3 S= \\
3 D= \\
1 D= \\
3 P= \\
1 P=\end{array}$} & $\begin{array}{l}{ }^{4} S \\
{ }^{4} S\end{array}$ & $\begin{array}{l}2 D \\
2 D\end{array}$ & \multicolumn{2}{|c|}{$\begin{array}{rl} & +34 P \\
+1 / 3 & 4 P+8 / 3{ }^{4} P \\
+4 / 3 & 4 P+1 / 62 P+3 / 2{ }^{2} D \\
& +3 / 22 P+3 / 22 D \\
+4 / 3 & 4 P+1 / 62 P+5 / 62 D+2 / 32 S \\
& +3 / 2{ }^{2} P+5 / 62 D+2 / 32 S\end{array}$} & \multicolumn{2}{|c|}{$\begin{array}{l}-3{ }^{3 P} \\
-3{ }^{3 P} \\
-3 / 2{ }^{3 P}-3 / 21 D \\
-3 / 2{ }^{3} P-3 / 21 D \\
-3 / 2{ }^{3} P-5 / 61 D-2 / 31 S \\
-3 / 2{ }^{3} P-5 / 61 D-2 / 31 S\end{array}$} & \multicolumn{2}{|c|}{ 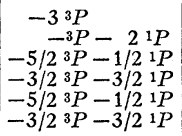 } & $\begin{array}{l}+32 P \\
+32 P \\
+32 P \\
+32 P \\
+32 P \\
+32 P\end{array}$ & $\begin{array}{l}+2 S \\
+2 S \\
+2 S \\
+2 S \\
+2 S \\
+2 S\end{array}$ \\
\hline & $s^{2} p$ & & $s p^{2}$ & & & $s p$ & $s^{2}$ & $p$ & & $s$ \\
\hline $\begin{array}{r}s^{2} p^{2}{ }^{3 P}= \\
1 D= \\
1 S=\end{array}$ & $\begin{array}{l}22 P \\
22 P \\
22 P\end{array}$ & $+4 / 34$ & $\begin{array}{r}+2 / 3^{2} P \\
+2{ }^{2} D+2{ }^{2} S\end{array}$ & ${ }^{-3 P}$ & $-1 S$ & $\begin{array}{l}-33^{3} P=1 P \\
-33^{3} P=1 P \\
-3{ }^{3} P-1 P\end{array}$ & $\begin{array}{l}-1 S \\
=1 S \\
=1 S\end{array}$ & $\begin{array}{l}+22 \\
+22 \\
+22\end{array}$ & & $\begin{array}{l}+22 S \\
+22 S \\
+22 S\end{array}$ \\
\hline
\end{tabular}

TABLE XI. Configuration $s p^{4}$.

\begin{tabular}{|c|c|c|c|c|c|c|c|c|c|c|c|}
\hline$\stackrel{s}{m_{s}}$ & $m_{s}$ & $m_{l}$ & $m_{s}$ & $m_{l}$ & $m_{s}{ }^{p}$ & $m_{l}$ & $m_{s}{ }^{p}$ & $m_{l}$ & $M_{S}$ & $M_{L}$ & Multiplets \\
\hline $1 / 2$ & $1 / 2$ & 1 & $1 / 2$ & 0 & $1 / 2$ & -1 & $-1 / 2$ & 1 & $3 / 2$ & 1 & ${ }^{4} P$ \\
\hline $1 / 2$ & $1 / 2$ & 1 & $1 / 2$ & 0 & $-1 / 2$ & 1 & $-1 / 2$ & 0 & $1 / 2$ & 2 & ${ }^{2} D$ \\
\hline $\begin{array}{r}-1 / 2 \\
1 / 2 \\
1 / 2\end{array}$ & $\begin{array}{l}1 / 2 \\
1 / 2 \\
1 / 2\end{array}$ & $\begin{array}{l}1 \\
1 \\
1\end{array}$ & $\begin{array}{l}1 / 2 \\
1 / 2 \\
1 / 2\end{array}$ & $\begin{array}{r}0 \\
0 \\
-1\end{array}$ & $\begin{array}{r}1 / 2 \\
-1 / 2 \\
-1 / 2\end{array}$ & $\begin{array}{r}-1 \\
1 \\
1\end{array}$ & $\begin{array}{l}-1 / 2 \\
-1 / 2 \\
-1 / 2\end{array}$ & $\begin{array}{r}1 \\
-1 \\
0\end{array}$ & $1 / 2$ & 1 & $\begin{array}{c}1 / 3{ }^{4} P+2 / 3^{2} P \\
1 / 3{ }^{4} P+1 / 6^{2} P+1 / 2^{2} D \\
\text { same }\end{array}$ \\
\hline $\begin{array}{r}-1 / 2 \\
1 / 2 \\
1 / 2 \\
1 / 2\end{array}$ & $\begin{array}{l}1 / 2 \\
1 / 2 \\
1 / 2 \\
1 / 2\end{array}$ & $\begin{array}{l}1 \\
1 \\
0 \\
1\end{array}$ & $\begin{array}{l}1 / 2 \\
1 / 2 \\
1 / 2 \\
1 / 2\end{array}$ & $\begin{array}{r}0 \\
0 \\
-1 \\
-1\end{array}$ & $\begin{array}{r}1 / 2 \\
-1 / 2 \\
-1 / 2 \\
-1 / 2\end{array}$ & $\begin{array}{r}-1 \\
0 \\
1 \\
1\end{array}$ & $\begin{array}{l}-1 / 2 \\
-1 / 2 \\
-1 / 2 \\
-1 / 2\end{array}$ & $\begin{array}{r}0 \\
-1 \\
0 \\
-1\end{array}$ & $1 / 2$ & 0 & $\begin{array}{r}1 / 3{ }^{4 P}+2 / 3{ }^{2} P \\
1 / 3{ }^{4} P+1 / 6{ }^{2} P+1 / 6{ }^{2} D+1 / 3{ }^{2} S \\
\text { same } \\
2 / 3{ }^{2} D+1 / 3{ }^{2} S\end{array}$ \\
\hline (A) & \multicolumn{2}{|c|}{ (B) } & \multicolumn{2}{|c|}{ (C) } & \multicolumn{2}{|c|}{$(D)$} & \multicolumn{2}{|c|}{$(E)$} & $M_{S}$ & $M_{L}$ & $(A B C D E)$ \\
\hline
\end{tabular}

Now it is possible to eliminate this state by using the relations for $p^{3}$ from Table VII. This can be done in a variety of ways since $p^{3}$ gives three multiplets ${ }^{3}$ but if we use the relation for ${ }^{4} S$ we find

$$
\begin{array}{r}
W_{2}{ }^{*}\left(p^{4}{ }^{3} P\right)=1 / 3\left(p^{3}{ }^{4} S^{*}\right)+5 / 3\left(p^{3}{ }^{2} D^{*}\right) \\
+\left(p^{3}{ }^{2} P^{*}\right)-\left(p^{2}{ }^{3} P^{*}\right)-5 / 3\left(p^{2}{ }^{1} D^{*}\right) \\
-1 / 3\left(p^{2}{ }^{1} S^{*}\right) .
\end{array}
$$

This expression cannot be expected to be as accurate as (27) since (27) is a second approximation and we have obtained (29) by elimination with a first approximation. The accuracy of (29) will be about that of a first approximation relation such as those in Table VII. No table of the relations of this lower approximation is given, since they can be obtained directly from Tables VII and X by elimination. Furthermore, several relations can be obtained for each state and the most desirable one will depend upon the information available in the particular example at hand.

\section{Five, six and seven electrons}

In the set of five electron systems we consider the configurations $s p^{4}, p^{5}$ and $s^{2} p^{3}$. Table XI gives the individual electron states for $s p^{4}$ and the linear combination of the multiplets ${ }^{4} P,{ }^{2} P$, ${ }^{2} D$ and ${ }^{2} S$ which characterizes each state. Several states which give no additional information about the energies are omitted. The energy relations for the various states of $s p^{4}, p^{5}$ and $s^{2} p^{3}$ are given in the following equations.

$$
\begin{aligned}
& s p^{4} \quad W_{3}\left(s p^{4}{ }^{4} P\right)=\left(p^{4}{ }^{3} P\right)+\left(s p^{3}\right)\left[5 / 4{ }^{5} S+5 / 3{ }^{3} D+{ }^{3} P+1 / 12{ }^{3} S\right]-\left(p^{3}\right)\left[4 / 3{ }^{4} S+5 / 3{ }^{2} D+{ }^{2} P\right] \\
& -\left(s p^{2}\right)\left[11 / 3{ }^{4} P+1 / 3{ }^{2} P+5 / 3{ }^{2} D+1 / 3{ }^{2} S\right]+\left(p^{2}\right)\left[4{ }^{3} P+5 / 3{ }^{1} D+1 / 3{ }^{1} S\right] \\
& +(s p)\left[7 / 2{ }^{3} P+1 / 2{ }^{1} P\right]-4\left(p^{2} P\right)-\left(s^{2} S\right),
\end{aligned}
$$

If we use any of the other relations the resulting equarelative accuracy of these different expressions depends on tions, though different, are quite as good as (23). The 


$$
\begin{aligned}
& W_{3}\left(s p^{4}{ }^{2} P\right)=\left(p^{4}{ }^{3} P\right)+\left(s p^{3}\right)\left[4 / 3{ }^{3} S+5 / 12{ }^{3} D+5 / 4{ }^{1} D+1 / 4{ }^{3} P+3 / 4{ }^{1} P\right] \\
& -\left(p^{3}\right)\left[4 / 3{ }^{4} S+5 / 3{ }^{2} D+{ }^{2} P\right]-\left(s p^{2}\right)\left[2 / 3{ }^{4} P+10 / 3{ }^{2} P+5 / 3{ }^{2} D+1 / 3{ }^{2} S\right] \\
& +\left(p^{2}\right)\left[4{ }^{3} P+5 / 3{ }^{1} D+1 / 3{ }^{1} S\right]+(s p)\left[2{ }^{3} P+2{ }^{1} P\right]-4\left(p^{2} P\right)-\left(s^{2} S\right), \\
& W_{3}\left(s p^{4}{ }^{2} D\right)=\left(p^{4}{ }^{1} D\right)+\left(s p^{3}\right)\left[9 / 4{ }^{3} D+3 / 4{ }^{1} D+3 / 4{ }^{3} P+1 / 4{ }^{1} P\right]-\left(p^{3}\right)\left[3^{2} D+{ }^{2} P\right] \\
& -\left(s p^{2}\right)\left[2{ }^{4} P+{ }^{2} P+8 / 3{ }^{2} D+1 / 3{ }^{2} S\right]+\left(p^{2}\right)\left[3{ }^{3} P+8 / 3{ }^{1} D+1 / 3{ }^{1} S\right] \\
& +(s p)\left[3{ }^{3} P+{ }^{1} P\right]-4\left(p^{2} P\right)-\left(s^{2} S\right), \\
& W_{3}\left(s p^{4}{ }^{2} S\right)=\left(p^{4}{ }^{1} S\right)+\left(s p^{3}\right)\left[3{ }^{3} P+{ }^{1} P\right]-4\left(p^{3}{ }^{2} P\right)-\left(s p^{2}\right)\left[2{ }^{4} P+{ }^{2} P+5 / 3{ }^{2} D+4 / 3{ }^{2} S\right] \\
& +\left(p^{2}\right)\left[3{ }^{3} P+5 / 3{ }^{1} D+4 / 3{ }^{1} S\right]+(s p)\left[3{ }^{3} P+{ }^{1} P\right]-4\left(p^{2} P\right)-\left(s^{2} S\right), \\
& p^{5} \quad W_{3}\left(p^{5}{ }^{2} P\right)=\left(p^{4}\right)\left[3{ }^{3} P+5 / 3{ }^{1} D+1 / 3{ }^{1} S\right]-\left(p^{3}\right)\left[2{ }^{4} S+5{ }^{2} D+3{ }^{2} P\right] \\
& +\left(p^{2}\right)\left[6{ }^{3} P+10 / 3{ }^{1} D+2 / 3{ }^{1} S\right]-5\left(p^{2} P\right), \\
& s^{2} p^{3} \quad W_{3}\left(s^{2} p^{3}{ }^{4} S\right)=3\left(s^{2} p^{2}{ }^{3} P\right)+\left(s p^{3}\right)\left[5 / 4{ }^{5} S+3 / 4{ }^{3} S\right]-3\left(s^{2} p^{2} P\right)-\left(s p^{2}\right)\left[4{ }^{4} P+2{ }^{2} P\right] \\
& -\left(p^{3}{ }^{4} S\right)+\left(s^{2}{ }^{1} S\right)+(s p)\left[9 / 2{ }^{3} P+3 / 2{ }^{1} P\right]+3\left(p^{2}{ }^{3} P\right)-2\left(s^{2} S\right)-3\left(p^{2} P\right), \\
& W_{3}\left(s^{2} p^{3}{ }^{2} D\right)=\left(s^{2} p^{2}\right)\left[3 / 2{ }^{3} P+3 / 2{ }^{1} D\right]+\left(s p^{3}\right)\left[3 / 2{ }^{3} D+1 / 2{ }^{1} D\right]-3\left(s^{2} p^{2} P\right) \\
& -\left(s p^{2}\right)\left[2{ }^{4} P+3{ }^{2} D+{ }^{2} P\right]-\left(p^{3}{ }^{2} D\right)+\left(s^{2}{ }^{1} S\right)+(s p)\left[9 / 2{ }^{3} P+3 / 2{ }^{1} P\right] \\
& +\left(p^{2}\right)\left[3 / 2{ }^{3} P+3 / 2{ }^{1} D\right]-2\left(s^{2} S\right)-3\left(p^{2} P\right), \\
& W_{3}\left(s^{2} p^{3}{ }^{2} P\right)=\left(s^{2} p^{2}\right)\left[3 / 2{ }^{3} P+5 / 6{ }^{1} D+2 / 3{ }^{1} S\right]+\left(s p^{3}\right)\left[3 / 2{ }^{3} P+1 / 2{ }^{1} P\right]-3\left(s^{2} p^{2} P\right) \\
& -\left(s p^{2}\right)\left[2{ }^{4} P+{ }^{2} P+5 / 3{ }^{2} D+4 / 3{ }^{2} S\right]-\left(p^{3}{ }^{2} P\right)+\left(s^{2}{ }^{1} S\right) \\
& +(s p)\left[9 / 2{ }^{3} P+3 / 2{ }^{1} P\right]+\left(p^{2}\right)\left[3 / 2{ }^{3} P+5 / 6{ }^{1} D+2 / 3{ }^{1} S\right]-2\left(s^{2} S\right)-3\left(p^{2} P\right) .
\end{aligned}
$$

The lower approximations can be found also for these configurations but since they can be obtained in the straightforward way described above for $p^{4}$ they are not given here.

Of the six electron configurations we consider $s p^{5}, p^{6}$ and $s^{2} p^{4}$. The energies of the various multiplets of these in terms of the energies of the higher ions, with all energies measured to the core beneath, are given in the following relations.

$$
\begin{aligned}
s p^{5} \quad W_{4}\left(s p^{5}{ }^{3} P\right)= & \left(p^{5} P\right)+\left(s p^{4}\right)\left[8 / 3{ }^{4} P+1 / 3{ }^{2} P+5 / 3{ }^{2} D+1 / 3{ }^{2} S\right]-\left(p^{4}\right)\left[3{ }^{3} P+5 / 3{ }^{1} D+1 / 3{ }^{1} S\right] \\
& -\left(s p^{3}\right)\left[5 / 3{ }^{5} S+1 / 3{ }^{3} S+25 / 6{ }^{3} D+5 / 6{ }^{1} D+5 / 2{ }^{3} P+1 / 2{ }^{1} P\right] \\
& +\left(p^{3}\right)\left[2{ }^{4} S+5{ }^{2} D+3{ }^{2} P\right]+\left(s p^{2}\right)\left[14 / 3{ }^{4} P+4 / 3{ }^{2} P+10 / 3{ }^{2} D+2 / 3{ }^{2} S\right] \\
& -\left(p^{2}\right)\left[6{ }^{3} P+10 / 3{ }^{1} D+2 / 3{ }^{1} S\right]-(s p)\left[4{ }^{3} P+{ }^{1} P\right]+5\left(p^{2} P\right)+\left({ }^{2} S\right), \\
W_{4}\left(s p^{5}{ }^{1} P\right)= & \left(p^{5}{ }^{2} P\right)+\left(s p^{4}\right)\left[3{ }^{2} P+5 / 3{ }^{2} D+1 / 3{ }^{2} S\right]-\left(p^{4}\right)\left[3{ }^{3} P+5 / 3{ }^{1} D+1 / 3{ }^{1} S\right] \\
& -\left(s p^{3}\right)\left[2{ }^{3} S+5 / 2{ }^{3} D+5 / 2{ }^{1} D+3 / 2{ }^{3} P+3 / 2{ }^{1} P\right]+\left(p^{3}\right)\left[2{ }^{4} S+5{ }^{2} D+3{ }^{2} P\right] \\
& +\left(s p^{2}\right)\left[2{ }^{4} P+4{ }^{2} P+10 / 3{ }^{2} D+2 / 3{ }^{2} S\right]-\left(p^{2}\right)\left[6{ }^{3} P+10 / 3{ }^{1} D+2 / 3{ }^{1} S\right] \\
p^{6} \quad & -(s p)\left[3{ }^{3} P+2{ }^{1} P\right]+5\left(p{ }^{2} P\right)+\left(s^{2} S\right), \\
W_{4}\left(p^{6}{ }^{1} S\right)= & 6\left(p^{5}{ }^{2} P\right)-\left(p^{4}\right)\left[9{ }^{3} P+5{ }^{1} D+{ }^{1} S\right]+\left(p^{3}\right)\left[4{ }^{4} S+10{ }^{2} D+6{ }^{2} P\right] \\
& -\left(p^{2}\right)\left[9{ }^{3} P+5{ }^{1} D+{ }^{1} S\right]+6\left(p_{-}{ }^{2} P\right),
\end{aligned}
$$




$$
\begin{aligned}
& s^{2} p^{4} \quad W_{4}\left(s^{2} p^{4}{ }^{3} P\right)=\left(s^{2} p^{3}\right)\left[4 / 3{ }^{4} S+5 / 3{ }^{2} D+{ }^{2} P\right]+\left(s p^{4}\right)\left[4 / 3{ }^{4} P+2 / 3{ }^{2} P\right] \\
& -\left(p^{4}{ }^{3} P\right)-\left(s^{2} p^{2}\right)\left[4^{3} P+5 / 3{ }^{1} D+1 / 3{ }^{1} S\right]-\left(s p^{3}\right)\left[5 / 3{ }^{5} S+{ }^{3} S+5 / 2{ }^{3} D+5 / 6{ }^{1} D\right. \\
& \left.+3 / 2{ }^{3} P+1 / 2{ }^{1} P\right]+4\left(s^{2} p^{2} P\right)+\left(p^{3}\right)\left[4 / 3{ }^{4} S+5 / 3{ }^{2} D+{ }^{2} P\right] \\
& +\left(s p^{2}\right)\left[16 / 3{ }^{4} P+8 / 3{ }^{2} P+10 / 3{ }^{2} D+2 / 3{ }^{2} S\right]-\left(s^{2}{ }^{1} S\right)-\left(p^{2}\right)\left[4{ }^{3} P+5 / 3{ }^{1} D+1 / 3{ }^{1} S\right] \\
& -(s p)\left[6{ }^{3} P+2{ }^{1} P\right]+2\left(s^{2} S\right)+4\left(p^{2} P\right), \\
& W_{4}\left(s^{2} p^{4} 1 D\right)=\left(s^{2} p^{3}\right)\left[3^{2} D+{ }^{2} P\right]+2\left(s p^{4}{ }^{2} D\right)-\left(p^{4}{ }^{1} D\right)-\left(s^{2} p^{2}\right)\left[3{ }^{3} P+8 / 3{ }^{1} D+1 / 3{ }^{1} S\right] \\
& -\left(s p^{3}\right)\left[9 / 2{ }^{3} D+3 / 2{ }^{1} D+3 / 2{ }^{3} P+1 / 2{ }^{1} P\right]+4\left(s^{2} p^{2} P\right)+\left(p^{3}\right)\left[3^{2} D+{ }^{2} P\right] \\
& +\left(s p^{2}\right)\left[4{ }^{4} P+2{ }^{2} P+16 / 3{ }^{2} D+2 / 3{ }^{2} S\right]-\left(s^{2}{ }^{1} S\right)-\left(p^{2}\right)\left[3{ }^{3} P+8 / 3{ }^{1} D+1 / 3{ }^{1} S\right] \\
& -(s p)\left[6{ }^{3} P+2{ }^{1} P\right]+2\left(s^{2} S\right)+4\left(p^{2} P\right), \\
& W_{4}\left(s^{2} p^{4}{ }^{1} S\right)=4\left(s^{2} p^{32} P\right)+2\left(s p^{4}{ }^{2} S\right)-\left(p^{4}{ }^{1} S\right)-\left(s^{2} p^{2}\right)\left[3{ }^{3} P+5 / 3{ }^{1} D+4 / 3{ }^{1} S\right]-\left(s p^{3}\right)\left[6{ }^{3} P+2{ }^{1} P\right] \\
& +4\left(s^{2} p^{2} P\right)+4\left(p^{3}{ }^{2} P\right)+\left(s p^{2}\right)\left[4{ }^{4} P+2{ }^{2} P+10 / 3{ }^{2} D+8 / 3{ }^{2} S\right] \\
& -\left(s^{2}{ }^{1} S\right)-\left(p^{2}\right)\left[3{ }^{3} P+5 / 3{ }^{1} D+4 / 3{ }^{1} S\right]-(s p)\left[6{ }^{3} P+2{ }^{1} P\right]+2\left(s^{2} S\right)+4\left(p^{2} P\right) \text {. }
\end{aligned}
$$

For the configuration $\left(s^{2} p^{5}\right)$ we have

$$
\begin{aligned}
s^{2} p^{5} \quad W_{5}\left(s^{2} p^{5}{ }^{2} P\right)= & \left(s^{2} p^{4}\right)\left[3{ }^{3} P+5 / 3{ }^{1} D+1 / 3{ }^{1} S\right]+\left(s p^{5}\right)\left[3 / 2{ }^{3} P+1 / 2{ }^{1} P\right]-\left(s^{2} p^{3}\right)\left[2{ }^{4} S+5{ }^{2} D+3{ }^{2} P\right] \\
& -\left(s p^{4}\right)\left[4{ }^{4} P+2{ }^{2} P+10 / 3{ }^{2} D+2 / 3{ }^{2} S\right]-\left(p^{5}{ }^{2} P\right)+\left(s^{2} p^{2}\right)\left[6{ }^{3} P+10 / 3{ }^{2} D+2 / 3{ }^{2} P\right] \\
& +\left(s p^{3}\right)\left[5 / 2{ }^{5} S+3 / 2{ }^{3} S+15 / 2{ }^{3} D+5 / 2{ }^{1} D+9 / 2{ }^{3} P+3 / 2{ }^{1} P\right] \\
& +\left(p^{4}\right)\left[3{ }^{3} P+5 / 3{ }^{1} D+1 / 3{ }^{1} S\right]-5\left(s^{2} p^{2} P\right)-\left(s p^{2}\right)\left[8{ }^{4} P+4{ }^{2} P+20 / 3{ }^{2} D+4 / 3{ }^{2} S\right] \\
& -\left(p^{3}\right)\left[2{ }^{4} S+5{ }^{2} D+3{ }^{2} P\right]+\left(s^{2}{ }^{1} S\right)+(s p)\left[15 / 2{ }^{3} P+5 / 2{ }^{1} P\right] \\
& +\left(p^{2}\right)\left[6{ }^{3} P+10 / 3{ }^{1} D+2 / 3{ }^{1} S\right]-2\left({ }^{2} S\right)-5\left(p^{2} P\right) .
\end{aligned}
$$

\section{IIIb. Inter-Multiplet Separations}

Relations have been developed above to give the absolute values of energy states in terms of those of higher ions. It is frequently the case among known energy states that the absolute values of several ions in succession are not known. Still it is desirable to know the relative separations in such cases. These can be obtained easily and directly for all the configurations which are studied above by subtraction of relations for states of the same configuration. Due to the equality of the sums of the coefficients for each configuration which occurs, these difference relations can always be written in terms of the energy differences between multiplets in the higher ions.

It is also useful to have these difference relations in lower approximation. For example, the direct differences of the relations given above for $s p^{4}$ give the separation of two multiplets in terms of multiplet differences in $s p^{3}, p^{4}, s p^{2}, p^{3}, s p$ and $p^{2}$. A lower approximation can be found which gives the same separation in terms of the same differences in $s p^{3}, p^{4}, s p^{2}$ and $p^{3}$. We find the coefficients as in section IIb.

$$
W_{\mathbf{3}}{ }^{\prime}(A B C D E)=2 / 3 \sum W(\alpha \beta \gamma \delta)-1 / 3 \sum W(\alpha \beta \gamma)+1 / 3 \sum W(\alpha) .
$$

If we subtract two such expressions for different multiplets of the same configuration we find that the actual differences are the same as in the next higher approximation but all are multiplied by the coefficients in (30). The difference relations for the various configurations discussed above are collected below. Since the differences are all given from the lowest level of the configuration any other difference can be obtained by one subtraction. Some of the other differences give much simpler relations. 
$p^{3} \quad\left(p^{3}\right)\left[{ }^{4} S-{ }^{2} D\right]=\left(p^{2}\right) 3 / 2\left({ }^{3} P-{ }^{1} D\right)$, $\left(p^{3}\right)\left[{ }^{4} S-{ }^{2} P\right]=\left(p^{2}\right)\left[5 / 6\left({ }^{3} P-{ }^{1} D\right)+2 / 3\left({ }^{3} P-{ }^{1} S\right)\right]$,

$s p^{2} \quad\left(s p^{2}\right)\left[{ }^{4} P-{ }^{2} P\right]=(s p) 3 / 2\left[{ }^{3} P-{ }^{1} P\right]$, $\left(s p^{2}\right)\left[{ }^{4} P-{ }^{2} D\right]=\left(p^{2}\right)\left[{ }^{3} P-{ }^{1} D\right]+(s p) 1 / 2\left[{ }^{3} P-{ }^{1} P\right]$, $\left(s p^{2}\right)\left[{ }^{4} P-{ }^{2} S\right]=\left(p^{2}\right)\left[{ }^{3} P-{ }^{1} S\right]+(s p) 1 / 2\left[{ }^{3} P-{ }^{1} P\right]$

$p^{4} \quad\left(p^{4}\right)\left[{ }^{3} P-{ }^{1} D\right]=\left(p^{3}\right) 4 / 3\left[{ }^{4} S-{ }^{2} D\right]-\left(p^{2}\right)\left[{ }^{3} P-{ }^{1} D\right]$, $\left(p^{4}\right)\left[{ }^{3} P-{ }^{1} S\right]=\left(p^{3}\right)\left[4 / 3\left({ }^{4} S-{ }^{2} P\right)+5 / 3\left({ }^{2} D-{ }^{2} P\right)\right]-\left(p^{2}\right)\left[{ }^{3} P-{ }^{1} S\right]$.

Next lower approximation:

$$
\begin{aligned}
& \left(p^{4}\right)\left[{ }^{3} P-{ }^{1} D\right]=1 / 2\left(p^{3}\right) 4 / 3\left[{ }^{4} S-{ }^{2} D\right], \\
& \left(p^{4}\right)\left[{ }^{3} P-{ }^{1} S\right]=1 / 2\left(p^{3}\right)\left[4 / 3\left({ }^{4} S-{ }^{2} P\right)+5 / 3\left({ }^{2} D-{ }^{2} P\right)\right] \text {, } \\
& s p^{3} \quad\left(s p^{3}\right)\left[{ }^{5} S-{ }^{3} S\right]=\left(s p^{2}\right) 8 / 3\left[{ }^{4} P-{ }^{2} P\right]-(s p) 2\left[{ }^{3} P-{ }^{1} P\right] \text {, } \\
& \left(s p^{3}\right)\left[{ }^{5} S-{ }^{3} D\right]=\left(p^{3}\right)\left[{ }^{4} S-{ }^{2} D\right]+\left(s p^{2}\right)\left[1 / 6\left({ }^{4} P-{ }^{2} P\right)+3 / 2\left({ }^{4} P-{ }^{2} D\right)\right] \\
& -\left(p^{2}\right) 3 / 2\left[{ }^{3} P-{ }^{1} D\right]-(s p) 1 / 2\left[{ }^{3} P-{ }^{1} P\right], \\
& \left(s p^{3}\right)\left[{ }^{5} S-{ }^{1} D\right]=\left(p^{3}\right)\left[{ }^{4} S-{ }^{2} D\right]+\left(s p^{2}\right)\left[3 / 2\left({ }^{4} P-{ }^{2} P\right)+3 / 2\left({ }^{4} P-{ }^{2} D\right)\right] \\
& -\left(p^{2}\right) 3 / 2\left[{ }^{3} P-{ }^{1} D\right]-(s p) 3 / 2\left[{ }^{3} P-{ }^{1} P\right], \\
& \left(s p^{3}\right)\left[{ }^{5} S-{ }^{3} P\right]=\left(p^{3}\right)\left[{ }^{4} S-{ }^{2} P\right]+\left(s p^{2}\right)\left[1 / 6\left({ }^{4} P-{ }^{2} P\right)+5 / 6\left({ }^{4} P-{ }^{2} D\right)+2 / 3\left({ }^{4} P-{ }^{2} S\right)\right] \\
& -\left(p^{2}\right)\left[5 / 6\left({ }^{3} P-{ }^{1} D\right)+2 / 3\left({ }^{3} P-{ }^{1} S\right)\right]-(s p) 1 / 2\left[{ }^{3} P-{ }^{1} P\right], \\
& \left(s p^{3}\right)\left[{ }^{5} S-{ }^{1} P\right]=\left(p^{3}\right)\left[{ }^{4} S-{ }^{2} P\right]+\left(s p^{2}\right)\left[3 / 2\left({ }^{4} P-{ }^{2} P\right)+5 / 6\left({ }^{4} P-{ }^{2} D\right)+2 / 3\left({ }^{4} P-{ }^{2} S\right)\right] \\
& -\left(p^{2}\right)\left[5 / 6\left({ }^{3} P-{ }^{1} D\right)+2 / 3\left({ }^{3} P-{ }^{1} S\right)\right]-(s p) 3 / 2\left[{ }^{3} P-{ }^{1} P\right] .
\end{aligned}
$$

The next lower approximation is obtained from the above by dropping those terms in two electrons $\left(s p\right.$ and $\left.p^{2}\right)$ and multiplying the terms in three electrons by a factor $1 / 2$. For example, for ${ }^{5} S-{ }^{3} D$ we have

$$
\left(s p^{3}\right)\left[{ }^{5} S-{ }^{3} D\right]=1 / 2\left(p^{3}\right)\left[{ }^{4} S-{ }^{2} D\right]+1 / 2\left(s p^{2}\right)\left[1 / 6\left({ }^{4} P-{ }^{2} P\right)+3 / 2\left({ }^{4} P-{ }^{2} D\right)\right],
$$

$s^{2} p^{2} \quad\left(s^{2} p^{2}\right)\left[{ }^{3} P-{ }^{1} D\right]=\left(s p^{2}\right)\left[4 / 3\left({ }^{4} P-{ }^{2} D\right)+2 / 3\left({ }^{2} P-{ }^{2} D\right)\right]-\left(p^{2}\right)\left[{ }^{3} P-{ }^{1} D\right]$,

$$
\left(s^{2} p^{2}\right)\left[{ }^{3} P-{ }^{1} S\right]=\left(s p^{2}\right)\left[4 / 3\left({ }^{4} P-{ }^{2} S\right)+2 / 3\left({ }^{2} P-{ }^{2} S\right)\right]-\left(p^{2}\right)\left[{ }^{3} P-{ }^{1} S\right] .
$$

For the next lower approximation drop the terms in $p^{2}$ and multiply those in $s p^{2}$ by $1 / 2$.

$$
\begin{aligned}
s p^{4} \quad\left(s p^{4}\right)\left[{ }^{4} P-{ }^{2} P\right]= & \left(s p^{3}\right)\left[5 / 4\left({ }^{5} S-{ }^{3} S\right)+5 / 4\left({ }^{3} D-{ }^{1} D\right)+3 / 4\left({ }^{3} P-{ }^{1} P\right)\right] \\
& -\left(s p^{2}\right) 3\left[{ }^{4} P-{ }^{2} P\right]+(s p) 3 / 2\left[{ }^{3} P-{ }^{1} P\right], \\
\left(s p^{4}\right)\left[{ }^{4} P-{ }^{2} D\right]= & \left(p^{4}\right)\left[{ }^{3} P-{ }^{1} D\right]+\left(s p^{3}\right)\left[1 / 4\left({ }^{3} P-{ }^{1} P\right)+1 / 12\left({ }^{3} S-{ }^{3} D\right)+3 / 4\left({ }^{5} S-{ }^{1} D\right)\right. \\
& \left.+1 / 2\left({ }^{5} S-{ }^{3} D\right)\right]-\left(p^{3}\right) 4 / 3\left[{ }^{4} S-{ }^{2} D\right]-\left(s p^{2}\right)\left[\left({ }^{4} P-{ }^{2} D\right)+2 / 3\left({ }^{4} P-{ }^{2} P\right)\right] \\
& +\left(p^{2}\right)\left[{ }^{3} P-{ }^{1} D\right]+(s p) 1 / 2\left[{ }^{3} P-{ }^{1} P\right] \\
\left(s p^{4}\right)\left[{ }^{4} P-{ }^{2} S\right]= & \left(p^{4}\right)\left[{ }^{3} P-{ }^{1} S\right]+\left(s p^{3}\right)\left[5 / 3\left({ }^{3} D-{ }^{3} P\right)+1 / 3\left({ }^{5} S-{ }^{3} P\right)+11 / 12\left({ }^{5} S-1 P\right)\right. \\
& \left.+1 / 12\left({ }^{3} S-{ }^{1} P\right)\right]-\left(p^{3}\right)\left[4 / 3\left({ }^{4} S-{ }^{2} P\right)+5 / 3\left({ }^{2} D-{ }^{2} P\right)\right] \\
& -\left(s p^{2}\right)\left[2 / 3\left({ }^{4} P-{ }^{2} P\right)+\left({ }^{4} P-{ }^{2} S\right)\right]+\left(p^{2}\right)\left[{ }^{3} P-{ }^{1} S\right]+(s p) 1 / 2\left[{ }^{3} P-{ }^{1} P\right] .
\end{aligned}
$$


To obtain the next lower approximation drop the terms in $s p$ and $p^{2}$ and multiply those in $p^{4}$ and $s p^{3}$ by $2 / 3$ and those in $p^{3}$ and $s p^{2}$ by $1 / 3$. For the lowest approximation drop the terms in $p^{3}$ and $s p^{2}$ as well and multiply those in $p^{4}$ and $s p^{3}$ by $1 / 3$.

$$
\begin{aligned}
& s^{2} p^{3} \quad\left(s^{2} p^{3}\right)\left[{ }^{4} S-{ }^{2} D\right]=\left(s^{2} p^{2}\right) 3 / 2\left[{ }^{3} P-{ }^{1} D\right]+\left(s p^{3}\right)\left[5 / 4\left({ }^{5} S-{ }^{3} D\right)+1 / 4\left({ }^{3} S-{ }^{3} D\right)+1 / 2\left({ }^{3} S-{ }^{1} D\right)\right] \\
& -\left(s p^{2}\right)\left[2\left({ }^{4} P-{ }^{2} D\right)+\left({ }^{2} P-{ }^{2} D\right)\right]-\left(p^{3}\right)\left[{ }^{4} S-{ }^{2} D\right]+\left(p^{2}\right) 3 / 2\left[{ }^{3} P-{ }^{1} D\right], \\
& \left(s^{2} p^{3}\right)\left[{ }^{4} S-{ }^{2} P\right]=\left(s^{2} p^{2}\right)\left[5 / 6\left({ }^{3} P-{ }^{1} D\right)+2 / 3\left({ }^{3} P-{ }^{1} S\right)\right]+\left(s p^{3}\right)\left[5 / 4\left({ }^{5} S-{ }^{3} P\right)+1 / 4\left({ }^{3} S-{ }^{3} P\right)\right. \\
& \left.+1 / 2\left({ }^{3} S-{ }^{1} P\right)\right]-\left(s p^{2}\right)\left[5 / 3\left({ }^{4} P-{ }^{2} D\right)+1 / 3\left({ }^{4} P-{ }^{2} S\right)+\left({ }^{2} P-{ }^{2} S\right)\right] \\
& -\left(p^{3}\right)\left[{ }^{4} S-{ }^{2} P\right]+\left(p^{2}\right)\left[5 / 6\left({ }^{3} P-{ }^{1} D\right)+2 / 3\left({ }^{3} P-{ }^{1} S\right)\right] .
\end{aligned}
$$

Follow the same procedure as for $s p^{4}$ to obtain the lower approximations.

$$
\begin{aligned}
s p^{5} \quad\left(s p^{5}\right)\left[{ }^{3} P-{ }^{1} P\right]=\left(s p^{4}\right) 8 / 3\left[{ }^{4} P-{ }^{2} P\right]-\left(s p^{3}\right)\left[5 / 3\left({ }^{5} S-{ }^{3} S\right)+5 / 3\right. & \left.\left({ }^{3} D-{ }^{1} D\right)+\left({ }^{3} P-{ }^{1} P\right)\right] \\
& +\left(s p^{2}\right) 8 / 3\left[{ }^{4} P-{ }^{2} P\right]-(s p)\left[{ }^{3} P-{ }^{1} P\right] .
\end{aligned}
$$

To obtain the next lower approximation drop the two electron terms and multiply all five electron terms by $3 / 4$, all four electron terms by $1 / 2$ and all three electron terms by $1 / 4$. For the next approximation drop the three electron terms as well and multiply the five electron terms by $1 / 2$ and the four electron terms by $1 / 6$. For the lowest approximation drop the four electron terms also and multiply the five electron terms by $1 / 4$.

$$
\begin{aligned}
s^{2} p^{4} \quad\left(s^{2} p^{4}\right)\left[{ }^{3} P-{ }^{1} D\right]= & \left(s^{2} p^{3}\right) 4 / 3\left[{ }^{4} S-{ }^{2} D\right]+\left(s p^{4}\right)\left[4 / 3\left({ }^{4} P-{ }^{2} D\right)+2 / 3\left({ }^{2} P-{ }^{2} D\right)\right]-\left(p^{4}\right)\left[{ }^{3} P-{ }^{1} D\right] \\
& -\left(s^{2} p^{2}\right)\left[{ }^{3} P-{ }^{1} D\right]-\left(s p^{3}\right)\left[5 / 3\left({ }^{5} S-{ }^{3} D\right)+1 / 3\left({ }^{3} S-{ }^{3} D\right)+2 / 3\left({ }^{3} S-{ }^{1} D\right)\right] \\
& +\left(p^{3}\right) 4 / 3\left[{ }^{4} S-{ }^{2} D\right]+\left(s p^{2}\right)\left[4 / 3\left({ }^{4} P-{ }^{2} D\right)+2 / 3\left({ }^{2} P-{ }^{2} D\right)\right]-\left(p^{2}\right)\left[{ }^{3} P-{ }^{1} D\right], \\
\left(s^{2} p^{4}\right)\left[{ }^{3} P-{ }^{1} S\right]= & \left(s^{2} p^{3}\right)\left[4 / 3\left({ }^{4} S-{ }^{2} P\right)+5 / 3\left({ }^{2} D-{ }^{2} P\right)\right]+\left(s p^{4}\right)\left[4 / 3\left({ }^{4} P-{ }^{2} S\right)+2 / 3\left({ }^{2} P-{ }^{2} S\right)\right] \\
& -\left(p^{4}\right)\left[{ }^{3} P-{ }^{1} S\right]-\left(s^{2} p^{2}\right)\left[{ }^{3} P-{ }^{1} S\right]-\left(s p^{3}\right)\left[5 / 3\left({ }^{5} S-{ }^{3} P\right)+\left({ }^{3} S-{ }^{3} P\right)\right. \\
& \left.+3 / 2\left({ }^{3} D-{ }^{1} P\right)+\left({ }^{3} D-{ }^{3} P\right)+5 / 6\left({ }^{1} D-{ }^{3} P\right)\right]+\left(p^{3}\right)\left[4 / 3\left({ }^{4} S-{ }^{2} P\right)+5 / 3\left({ }^{2} D-{ }^{2} P\right)\right] \\
& +\left(s p^{2}\right)\left[4 / 3\left({ }^{4} P-{ }^{2} S\right]+2 / 3\left({ }^{2} P-{ }^{2} S\right)\right]-\left(p^{2}\right)\left[{ }^{3} P-{ }^{1} S\right] .
\end{aligned}
$$

To obtain the lower approximations follow the same procedure as for $s p^{5}$.

\section{Examples}

(a) The relations developed in the preceding section IIIa express the energy of a given state of an atom or ion in terms of the energy states of the higher ions. In these relations we may use the values of the calculated energies ${ }^{4}$ or we may use experimentally known states and from them determine other states. It is the latter application which is most useful since the energies have, for the greater part, been calculated only to the first order by the perturbation method and in general do not agree very well with the observed

\footnotetext{
${ }^{4}$ For example, if the Slater relations are known for two electrons they can be found for several electrons by using the relations which give the many electron energies in terms of those of two electrons.
}

states. The examples considered here will be confined to the elements $\mathrm{O}, \mathrm{N}$ and $\mathrm{C}$ largely because the beautiful work of Edlén ${ }^{5}$ has made the energy states of these elements so fully and accurately known. Table XII gives the energies of various states of $\mathrm{O}, \mathrm{N}$ and $\mathrm{C}$ relative to the $1 s^{2}$ core, as taken from the work of Edlén. The spin-orbit separations have been reduced to the center of gravity.

\section{Three electrons}

Let us consider the state $2 s 2 p^{2}{ }^{2} P$. From Table VII we find the following relation

${ }^{5}$ B. Edlén, Zeits. f. Physik 84, 746 (1933). This is a condensed work. For complete information see Nova Acta Regiae Societatis Scientiarum Upsaliensis, Series IV, Vol. 9, No. 6 . 
ATOMIC ENERGY RELATIONS

TABLE XII. Energies to the $1 s^{2}$ shell for oxygen, nitrogen and carbon.

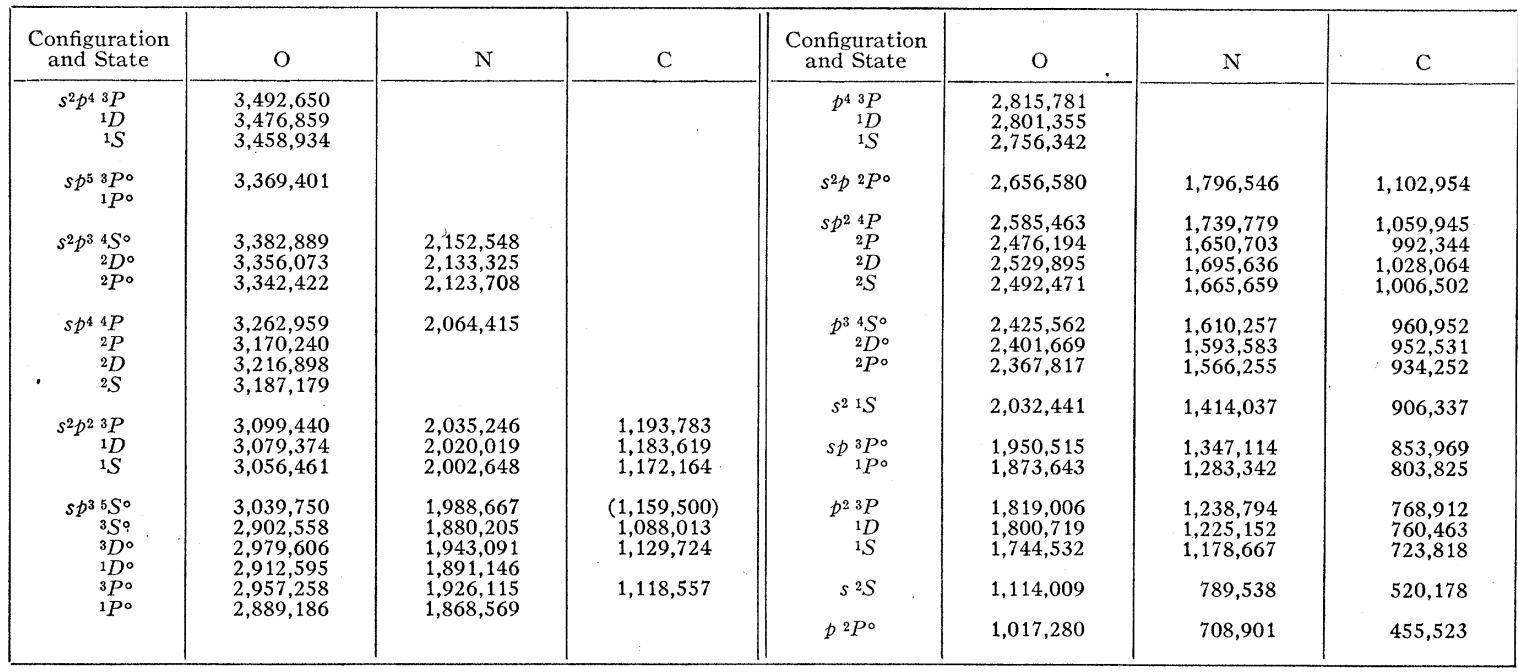

TABLE XIII. Three electron configurations. Absolute values to normal state of next ion.

\begin{tabular}{|c|c|c|c|c|c|c|c|c|c|}
\hline \multirow{2}{*}{$\begin{array}{l}\text { Configuration } \\
\text { and State }\end{array}$} & \multicolumn{3}{|c|}{$\mathrm{O}$} & \multicolumn{3}{|c|}{$\mathrm{N}$} & \multicolumn{3}{|c|}{$\mathrm{C}$} \\
\hline & Calc. & Obs. & Diff. & Calc. & Obs. & Diff. & Calc. & Obs. & Diff. \\
\hline $2 s^{2} 2 p{ }^{2} P$ & 617,295 & 624,139 & 6,844 & 374,365 & 382,509 & 8,144 & 187,067 & 196,617 & 9,550 \\
\hline $\begin{array}{r}2 s 2 p^{2}{ }^{4} P \\
2 P \\
{ }^{2} D \\
{ }^{2} S\end{array}$ & $\begin{array}{l}539,026 \\
423,717 \\
482,302 \\
426,115\end{array}$ & $\begin{array}{l}553,022 \\
443,753 \\
497,454 \\
460,030\end{array}$ & $\begin{array}{l}13,996 \\
20,036 \\
15,152 \\
33,915 \\
\end{array}$ & $\begin{array}{l}311,645 \\
215,987 \\
266,117 \\
219,632\end{array}$ & $\begin{array}{l}325,742 \\
236,666 \\
281,599 \\
251,622\end{array}$ & $\begin{array}{l}14,097 \\
20,679 \\
15,482 \\
31,990\end{array}$ & $\begin{array}{r}139,289 \\
64,073 \\
105,768 \\
69,123\end{array}$ & $\begin{array}{r}153,608 \\
86,007 \\
121,728 \\
100,165\end{array}$ & $\begin{array}{l}14,319 \\
21,934 \\
15,960 \\
31,042\end{array}$ \\
\hline $\begin{array}{r}2 p^{3} 4 S \\
2 D \\
2 P\end{array}$ & $\begin{array}{l}372,737 \\
345,305 \\
307,850\end{array}$ & $\begin{array}{l}393,121 \\
369,228 \\
335,376\end{array}$ & $\begin{array}{l}20,384 \\
23,923 \\
27,524\end{array}$ & $\begin{array}{l}175,642 \\
155,179 \\
124,189\end{array}$ & $\begin{array}{l}196,220 \\
179,546 \\
152,218\end{array}$ & $\begin{array}{l}20,578 \\
24,367 \\
28,029\end{array}$ & $\begin{array}{r}33,830 \\
21,155 \\
-3,272\end{array}$ & $\begin{array}{l}54,615 \\
46,194 \\
27,915\end{array}$ & $\begin{array}{l}20,785 \\
24,039 \\
31,187\end{array}$ \\
\hline $\begin{array}{r}\left(2 s^{2}\right) 2 p^{3}{ }^{4} S \\
2 D \\
2 P\end{array}$ & $\begin{array}{l}261,376 \\
231,271 \\
216,001\end{array}$ & $\begin{array}{l}283,244 \\
256,428 \\
242,777\end{array}$ & $\begin{array}{l}21,868 \\
25,157 \\
26,776\end{array}$ & $\begin{array}{l}94,803 \\
71,961 \\
60,382\end{array}$ & $\begin{array}{r}117,214 \\
97,991 \\
88,374\end{array}$ & $\begin{array}{l}22,411 \\
26,030 \\
27,992\end{array}$ & & & \\
\hline
\end{tabular}

$\left(s p^{2}\right)^{2} P=\left(p^{2}\right){ }^{3} P+(s p)\left[1 / 2{ }^{3} P+3 / 2{ }^{1} P\right]$

$$
-2(p)^{2} P-(s)^{2} S \text {. }
$$

If we use this relation to determine the state $\left(s p^{2}\right)^{2} P$, then we insert the correct energies on the right side from Table XII. For O IV we find $\left(s p^{2}\right)^{2} P=2,456,158 \mathrm{~cm}^{-1}$ with respect to the $1 s^{2}$ shell while the observed energy is $2,476,194$ $\mathrm{cm}^{-1}$. The discrepancy between the two is 20,036 $\mathrm{cm}^{-1}$. This appears to be a rather small error compared to the total energy to the shell beneath and it is even fairly small compared to the interaction energy of the three electrons, 672,375 $\mathrm{cm}^{-1}$. Under the usual criteria for a perturbation problem this would be declared to be a good approximation. Unfortunately, however, we are seldom interested in the total energy but rather in the "absolute energy" to the normal state of the next ion. This absolute value calculated is 423,717 and observed $443,753 \mathrm{~cm}^{-1}$. This error in the calculated value is due to the omission of the triple energy and we may therefore expect the discrepancy for the three electron cases to be greater than for more electrons where the triple energies are included. The results for configurations $2 s^{2} 2 p, 2 s 2 p^{2}, 2 p^{3}$ and $\left(2 s^{2}\right) 2 p^{3}$ (i.e., $2 p^{3}$ outside a closed $2 s^{2}$ shell), giving the calculated and observed absolute values to the normal state of the next ion and their differences, are presented in Table XIII. The difference between the calculated and observed values (the triple energy) for a given state is nearly constant ${ }^{6}$ with

${ }^{6}$ The regularities of the pair and triple energies as well as the total electrostatic interaction as a function of $Z$ will be discussed in a later paper. 
TABLE XIV. The four electron configurations. Absolute values to normal state of next ion.

\begin{tabular}{|c|c|c|c|c|c|c|c|c|c|}
\hline \multirow{2}{*}{$\begin{array}{l}\text { Configuration } \\
\text { and State }\end{array}$} & \multicolumn{3}{|c|}{$\mathrm{O}$} & \multicolumn{3}{|c|}{$\mathrm{N}$} & \multicolumn{3}{|c|}{$\mathrm{C}$} \\
\hline & Calc. & Obs. & Obs.-Calc. & Calc. & Obs. & Obs.-Calc. & Calc. & Obs. & Obs.-Calc. \\
\hline $\begin{array}{r}2 s^{2} 2 p^{2}{ }^{3} P \\
1 D \\
1 S\end{array}$ & $\begin{array}{l}440,345 \\
420,342 \\
401,681\end{array}$ & $\begin{array}{l}442,602 \\
422,536 \\
399,623\end{array}$ & $\begin{array}{r}2257 \\
2194 \\
-2058\end{array}$ & $\begin{array}{l}235,965 \\
220,707 \\
207,238\end{array}$ & $\begin{array}{l}238,584 \\
223,357 \\
205,986\end{array}$ & $\begin{array}{r}2619 \\
2650 \\
-1252\end{array}$ & $\begin{array}{l}88,155 \\
77,910 \\
71,431\end{array}$ & $\begin{array}{l}90,786 \\
80,622 \\
69,167\end{array}$ & $\begin{array}{r}2631 \\
2712 \\
-2264\end{array}$ \\
\hline $\begin{array}{r}2 s 2 p^{3} 5 S \\
3 S \\
3 D \\
3 D \\
1 D \\
3 P \\
1 P\end{array}$ & $\begin{array}{l}382,399 \\
244,756 \\
322,816 \\
253,993 \\
301,469 \\
232,648\end{array}$ & $\begin{array}{l}382,912 \\
245,720 \\
322,768 \\
255,757 \\
300,420 \\
232,348\end{array}$ & $\begin{array}{r}513 \\
964 \\
-48 \\
1764 \\
-1049 \\
-300\end{array}$ & $\begin{array}{r}191,449 \\
81,456 \\
146,062 \\
91,066 \\
129,740 \\
74,744\end{array}$ & $\begin{array}{r}192,005 \\
83,543 \\
146,429 \\
94,484 \\
129,453 \\
71,907\end{array}$ & $\begin{array}{r}556 \\
2087 \\
367 \\
3418 \\
-287 \\
-2837\end{array}$ & $\begin{array}{r}55,894 \\
-24,412 \\
26,053 \\
-14,027 \\
17,827 \\
-22,326\end{array}$ & $\begin{array}{r}-\overline{14,984} \\
26,727 \\
15,560 \\
\end{array}$ & $\begin{array}{r}9428 \\
\quad 674 \\
-2267 \\
\end{array}$ \\
\hline $\begin{array}{r}2 p^{4} 3 P \\
1 D \\
1 S\end{array}$ & $\begin{array}{r}158,228 \\
144,657 \\
99,288\end{array}$ & $\begin{array}{r}158,943 \\
144,517 \\
99,504\end{array}$ & $\begin{array}{r}715 \\
-140 \\
216 \\
\end{array}$ & $\begin{array}{r}18,191 \\
9,603 \\
-25,896 \\
\end{array}$ & $\bar{E}$ & & & & \\
\hline $\begin{array}{r}\left(2 s^{2}\right) 2 p^{4} 3 P \\
1 D \\
1 S\end{array}$ & $\begin{array}{r}108,516 \\
92,827 \\
74,787\end{array}$ & $\begin{array}{r}109,761 \\
93,970 \\
76,045\end{array}$ & $\begin{array}{l}1245 \\
1143 \\
1258\end{array}$ & & & & & & \\
\hline
\end{tabular}

$Z$. Thus if an adjacent element is known the actual correction (triple energy) can be extrapolated with considerable accuracy.

\section{Four electrons}

Since the triple energies are included in the relations for four electrons we expect them to agree better with the experimentally determined states and indeed this proves to be the case. Table XIV gives the absolute energies (to the normal state of the next ion) of the various multiplets of the configurations $2 s^{2} 2 p^{2}, 2 s 2 p^{3}$, $2 p^{4}$ and $\left(2 s^{2}\right) 2 p^{4}$ as determined from the relations developed above and as observed, together with their differences. The discrepancy for $s^{2} p^{2}$ in the absolute values is about $2500 \mathrm{~cm}^{-1}$. The relative value ${ }^{3} P-1 D$ is, however, within $100 \mathrm{~cm}^{-1}$ for all three elements. The ${ }^{1} D-{ }^{1} S$ separation has a larger discrepancy than the absolute values. It is interesting to note how the differences (observed minus calculated) decrease with increasing $Z$, with the exception of ${ }^{1} S$ for $N$. The configuration $s p^{3}$ fits better than $s^{2} p^{2}$ except for one or two multiplets in $N$ and $\mathrm{C}$ which are not far from the ionization limit. In general we can expect these relations to hold best for the low terms of high multiplicity since the higher orders are not as important. ${ }^{7}$ In $\mathrm{C}$, the $s p^{3}{ }^{5} S$ state, which is important as the lowest tetravalent state, has been predicted at $55,894 \mathrm{~cm}^{-1}$.

${ }^{7}$ It is possible to apply these relations to the determination of the energy states of negative ions. The higher orders, however, may be important and the results therefore may not be very accurate. For example, one finds that $\mathrm{O}$ should have an electron affinity in the $2 p^{5}$ state of about $8000 \mathrm{~cm}^{-1}$ or about $1 \mathrm{e}$. volt, whereas Lozier (Washington meeting 1934) gives $2.2 \pm 0.2$ e. volts.
This is $34,994 \mathrm{~cm}^{-1}$ or 4.32 electron-volts above the normal state of $\mathrm{C}$. The multiplets of $p^{4}$ (O III) agree somewhat better than either of the other configurations and for $\left(2 s^{2}\right) 2 p^{4}$ (normal state of $\mathrm{O} \mathrm{I}$ ), the absolute values are all about $1200 \mathrm{~cm}^{-1}$ too small, thus making the separations check with a maximum error of about $100 \mathrm{~cm}^{-1}$. The error of only $15 \mathrm{~cm}^{-1}$ in the ${ }^{3} P-{ }^{1} S$ separation is certainly fortuitous.

\section{Five and six electrons}

The ordinary absolute values with respect to the normal state of the next ion, of the multiplets of configurations $2 s^{2} 2 p^{3}$ of $\mathrm{O}$ and $\mathrm{N}$ and of $2 s 2 p^{4}$ and $2 s^{2} 2 p^{4}$ of $\mathrm{O}$, determined by the methods above, the values obtained experimentally, and the differences between these are presented in Table XV. It has been pointed out

TABLE XV. Five and six electron configurations. Absolute values to normal state of next ion.

\begin{tabular}{|c|c|c|c|c|c|c|}
\hline \multirow{2}{*}{$\begin{array}{l}\text { Configuration } \\
\text { and State }\end{array}$} & \multicolumn{3}{|c|}{0} & \multicolumn{3}{|c|}{${ }^{\prime} \mathrm{N}$} \\
\hline & Calc. & Obs. & $\begin{array}{l}\text { Obs.- } \\
\text { Calc. }\end{array}$ & Calc. & Obs. & $\begin{array}{l}\text { Obs.- } \\
\text { Calc. }\end{array}$ \\
\hline $\begin{array}{r}2 s^{2} 2 p^{3}{ }^{4} S \\
2 D \\
2 P\end{array}$ & $\begin{array}{l}283,121 \\
256,019 \\
241,802\end{array}$ & $\begin{array}{l}283,244 \\
256,428 \\
242,777\end{array}$ & $\begin{array}{l}123 \\
409 \\
975\end{array}$ & $\begin{array}{r}117,640 \\
98,583 \\
86,558\end{array}$ & $\begin{array}{r}117,214 \\
97,991 \\
88,374\end{array}$ & $\begin{array}{r}-426 \\
-592 \\
1816\end{array}$ \\
\hline $\begin{array}{r}2 s 2 p^{4}{ }^{4 P} \\
2 P \\
2 D \\
2 S\end{array}$ & $\begin{array}{r}162,775 \\
68,964 \\
115,860 \\
85,804\end{array}$ & $\begin{array}{r}163,314 \\
70,595 \\
117,253 \\
87,534\end{array}$ & \begin{tabular}{r|}
539 \\
1631 \\
1393 \\
1730
\end{tabular} & & & \\
\hline $\begin{array}{r}2 s^{2} 2 p^{4} 3 P \\
1 D \\
1 S\end{array}$ & $\begin{array}{r}111,038 \\
95,472 \\
78,465\end{array}$ & $\begin{array}{r}109,761 \\
93,970 \\
76,045\end{array}$ & $\begin{array}{l}-1277 \\
-1502 \\
-2420\end{array}$ & & & \\
\hline
\end{tabular}

that the methods used here are more accurate the more electrons there are considered: thus $p^{4}$ is better than $p^{3}$. This does not go on indefinitely, however, since as more electrons are 
added the 0th approximation recedes further from the actual problem and higher orders become larger. The percentage error on the total energy measured to the closed shell beneath will continue to decrease but the actual error may not. There are also other perturbing effects due to spin-orbit interaction which become apparent in these higher approximations. The absolute energies calculated for $2 s^{2} 2 p^{4}$ of O I are enough greater than the energies obtained when only the $2 p^{4}$ group .was considered that the differences between the observed and calculated values have negative signs, and indeed the deviations are not as small as they are for $2 p^{4}$. This is a case in which the actual size of the first term not considered in the approximation is higher for $s^{2} p^{4}$ than for $p^{4}$, in spite of the fact that the former is a higher approximation.

The atomic energy relations developed here have been used in the examples given above to calculate the absolute energies of atoms and ions in terms of the energies of higher ions. The spectroscopist is generally interested in the reverse process and these relations can be used as well for this purpose. Let us consider $\left(2 s^{2}\right) 2 p^{4}$ of $\mathrm{O} I$ and suppose that we wish to find the absolute value of $2 s^{2} 2 p^{2} P$ in O IV. The energies of all the states which were used in the relations for $2 p^{4}$ were with respect to the $2 s^{2}$ shell but if we do not know the O IV states we would know the states of the lower ions only with respect to $2 s^{2} 2 p^{2} P_{\frac{1}{2}}$. Using energies to this limit (indicated by a prime) we can rewrite the relation for $\left(2 s^{2}\right) 2 p^{4}{ }^{3} P$ in the following way:

$$
\begin{gathered}
4\left(s^{2} p\right)^{2} P-3\left(s^{2} p\right)^{2} P_{\frac{1}{2}}=\left(s^{2} p\right)^{2} P-2 \Delta^{2} P=\left(s^{2} p^{4}\right){ }^{3} P^{\prime} \\
-\left(s^{2} p^{3}\right)\left[4 / 3{ }^{4} S^{\prime}+5 / 3^{2} D^{\prime}+{ }^{2} P^{\prime}\right] \\
+\left(s^{2} p^{2}\right)\left[4{ }^{3} P^{\prime}+5 / 3^{1} D^{\prime}+1 / 3{ }^{1} S^{\prime}\right],
\end{gathered}
$$

where $\Delta^{2} P$ is ${ }^{8}$ the separation ${ }^{2} P_{\frac{1}{2}}-{ }^{2} P_{1 \frac{1}{3}}$, assuming the interval rule. If we neglect this correction we find

$\left(s^{2} p\right)^{2} P: 624,611 \mathrm{~cm}^{-1}$, observed : 624,139 $\mathrm{cm}^{-1}$.

If we know the ${ }^{2} P$ separation (as we do here) we would find a value $\left(s^{2} p\right)^{2} P=625,384 \mathrm{~cm}^{-1}$ which does not agree so well. The error in the corrected absolute value (1245) is about the same as the error for the normal state of O I, but the percentage error is very much smaller due to the large ionization potential.

Let us consider the configuration $s^{2} p^{3}$. If we rewrite the relation for $s^{2} p^{3}{ }^{4} S$ so that the energies (indicated with a prime) are all measured to $(s)^{2} S_{\frac{1}{3}}$ for the case in which absolute values in the highest ion are unknown, we find

$$
\begin{array}{r}
3(p){ }^{2} P-2(s){ }^{2} S_{\frac{1}{3}}=(p)^{2} P-2 \nu\left({ }^{2} S_{\frac{1}{3}}-{ }^{2} P_{1 \frac{1}{2}}\right)+\Delta^{2} P \\
=-\left(s^{2} p^{3}\right){ }^{4} S^{\prime}+3\left(s^{2} p^{2}\right)^{3} P^{\prime}+\left(s p^{3}\right)\left[5 / 4{ }^{5} S^{\prime}+3 / 4{ }^{3} S^{\prime}\right] \\
-3\left(s^{2} p\right){ }^{2} P^{\prime}-\left(s p^{2}\right)\left[44^{4} P^{\prime}+2^{2} P^{\prime}\right]-\left(p^{3}\right){ }^{4} S^{\prime}+\left(s^{2}\right)^{1} S^{\prime} \\
+(s p)\left[9 / 2{ }^{3} P^{\prime}+3 / 2{ }^{1} P^{\prime}\right]+3\left(p^{2}\right)^{3} P^{\prime},
\end{array}
$$

${ }^{8}$ This complication is removed, of course, if $\Delta^{2} P$ is known. where $\nu\left({ }^{2} S_{\frac{1}{2}}-{ }^{2} P_{1 \frac{1}{2}}\right)$ is one of the resonance lines. Thus if we know the states in the lower ions and this one spectral line we can (neglecting $\Delta^{2} P$ ) immediately get the absolute values. For $\mathrm{O}$ we have

$$
\begin{aligned}
&(p){ }^{2} P-2 \nu\left({ }^{2} S_{\frac{1}{3}}-{ }^{2} P_{1 \frac{1}{3}}\right)+\Delta^{2} P=823,693 \mathrm{~cm}^{-1}, \\
& \nu_{\text {obs. }}=96,907.7 \mathrm{~cm}^{-1}, \quad(p){ }^{2} P+\Delta^{2} P=1,017,508 ; \\
& \text { observed : } 1,017,280 \mathrm{~cm}^{-1} .
\end{aligned}
$$

Using the observed $\Delta^{2} P$ we find

$$
\text { ( } p)^{2} P=1,016,975 \text {, error }: 305 \mathrm{~cm}^{-1} \text {. }
$$

If we did not know this one line it would still be possible to eliminate either $(s)^{2} S$ or $(p)^{2} P$ between relations for two different configurations, e.g., $s^{2} p^{3}$ and $s p^{4}$, and solve finally for both.

For the examples in $\mathrm{O}, \mathrm{N}$ and $\mathrm{C}$ considered here, only those states have been considered which involve electrons with total quantum number $n=2$. For these elements the states which have an electron with higher $n$ value such as $2 s^{2} 2 p^{2} 4 s$ are not suited to this method since the electrostatic interaction of the inner electrons may easily be larger than the energy of the outer electron in the central field. In such a case the multiplets which arise from the same state in the ion lie very close together. The separation of the different groups built on different states in the ion can be found directly from the distance of these basis states in the ion.

(b) In section III(b) it was shown that expressions could be found for the differences of energy states within a configuration, in terms of the differences found in higher ions. To use these expressions absolute values are not necessary. The various multiplet separations of $2 p^{3},\left(2 s^{2}\right) 2 p^{3}$ and $2 s 2 p^{2}$ determined from these relations and observed for the elements $\mathrm{O}, \mathrm{N}$ and $\mathrm{C}$, are presented, together with their differences, in Table XVI. The differences between the observed and calculated values are roughly constant with $Z$, as they were for the absolute values. The errors for the three electron configurations are rather large but their regularity for different $Z$ makes a much closer prediction possible if an adjacent element is known.

The differences calculated and observed for the four electron configurations $2 s^{2} 2 p^{2}, 2 s 2 p^{3}$, $2 p^{4}$ and $\left(2 s^{2}\right) 2 p^{4}$ are presented in Table XVII. For four electrons we have two approximations for the differences. The higher one (marked II) determines the separations in terms of differences in the three and two electron ions, and the lower one uses only the three electron ions. It is interesting to notice that certain separations [for example $\left.2 s^{2} 2 p^{2}\left({ }^{3} P-{ }^{1} D\right)\right]$ are quite close in the lower approximation while other differences $2 s^{2} 2 p^{2}\left({ }^{1} D-{ }^{1} S\right)$ are much more in error. The 
TABLE XVI. Three electron configurations. Inter-multiplet separations.

\begin{tabular}{|c|c|c|c|c|c|c|c|c|c|}
\hline \multirow{2}{*}{$\begin{array}{l}\text { Configuration } \\
\text { and States }\end{array}$} & \multicolumn{3}{|c|}{$\mathrm{O}$} & \multicolumn{3}{|c|}{$\mathrm{N}$} & \multicolumn{3}{|c|}{ C } \\
\hline & Calc. & Obs. & Obs.-Calc. & Calc. & Obs. & Obs.-Calc. & Calc. & Obs. & Obs.-Calc. \\
\hline $\begin{array}{r}2 p^{3}{ }^{4} S-2 D \\
2 D-2 P \\
4 S-2 P\end{array}$ & $\begin{array}{l}27,430 \\
37,458 \\
64,888\end{array}$ & $\begin{array}{l}23,893 \\
33,852 \\
57,745\end{array}$ & $\begin{array}{l}-3537 \\
-3606 \\
-7143\end{array}$ & $\begin{array}{l}20,463 \\
30,997 \\
51,460\end{array}$ & $\begin{array}{l}16,674 \\
27,328 \\
44,002\end{array}$ & $\begin{array}{l}-3789 \\
-3669 \\
-7458\end{array}$ & $\begin{array}{l}12,673 \\
24,430 \\
37,103\end{array}$ & $\begin{array}{r}8,421 \\
18,279 \\
26,700\end{array}$ & $\begin{array}{r}-4,252 \\
-6,151 \\
-10,403\end{array}$ \\
\hline $\begin{array}{r}\left(2 s^{2}\right) 2 p^{3}{ }^{4} S-2 D \\
2 D=2 P \\
{ }^{4} S-2 P\end{array}$ & $\begin{array}{l}30,099 \\
15,275 \\
45,374\end{array}$ & $\begin{array}{l}26,816 \\
13,651 \\
40,467\end{array}$ & $\begin{array}{l}-3283 \\
-1624 \\
-4907\end{array}$ & $\begin{array}{l}22,840 \\
11,581 \\
34,421\end{array}$ & $\begin{array}{r}19,223 \\
9,617 \\
28,840\end{array}$ & $\begin{array}{l}-3617 \\
-1964 \\
-5581\end{array}$ & & & \\
\hline $\begin{array}{r}2 s 2 p^{2}{ }^{4} P-2 P \\
4 P-2 D \\
2 D-2 P \\
2 D-2 S\end{array}$ & $\begin{array}{r}115,308 \\
56,723 \\
58,585 \\
56,187\end{array}$ & $\begin{array}{r}109,269 \\
55,568 \\
53,701 \\
37,424\end{array}$ & $\begin{array}{r}-6039 \\
-1155 \\
-4884 \\
-18,763\end{array}$ & $\begin{array}{l}95,658 \\
45,528 \\
50,130 \\
46,485\end{array}$ & $\begin{array}{l}89,076 \\
44,143 \\
44,933 \\
29,977\end{array}$ & $\begin{array}{r}-6582 \\
-1385 \\
-5197 \\
-16,508\end{array}$ & $\begin{array}{l}75,216 \\
33,521 \\
41,695 \\
36,645\end{array}$ & $\begin{array}{l}67,601 \\
31,881 \\
35,720 \\
21,562\end{array}$ & $\begin{array}{r}-7,615 \\
-1,640 \\
-5,975 \\
-15,083\end{array}$ \\
\hline
\end{tabular}

TABLE XVII. Four electron configurations. Inter-multiplet separations.

\begin{tabular}{|c|c|c|c|c|c|c|c|c|c|c|}
\hline \multirow{2}{*}{\multicolumn{2}{|c|}{$\begin{array}{l}\text { Configuration } \\
\text { and States }\end{array}$}} & \multicolumn{3}{|c|}{$\mathrm{O}$} & \multicolumn{3}{|c|}{$\mathrm{N}$} & \multicolumn{3}{|c|}{$\mathrm{C}$} \\
\hline & & Calc. & Obs. & Obs.-Calc. & Calc. & Obs. & Obs.-Calc. & Calc. & Obs. & Obs.-Calc. \\
\hline & $\begin{array}{r}2 s^{2} 2 p^{2}{ }^{3} P-1 D \\
1 D-1 S \\
1 D-1 S \\
{ }^{3} P-1 S \\
\end{array}$ & $\begin{array}{l}20,002 \\
18,661 \\
38,663 \\
\end{array}$ & $\begin{array}{l}20,066 \\
22,913 \\
42,979 \\
\end{array}$ & $\begin{array}{r}64 \\
4252 \\
4316 \\
\end{array}$ & $\begin{array}{l}15,260 \\
13,469 \\
28,729 \\
\end{array}$ & $\begin{array}{l}15,227 \\
17,371 \\
32,598 \\
\end{array}$ & $\begin{array}{l}-33 \\
3902 \\
3869 \\
\end{array}$ & $\begin{array}{r}10,245 \\
6,479 \\
16,724 \\
\end{array}$ & $\begin{array}{l}10,164 \\
11,455 \\
21,619\end{array}$ & $\begin{array}{l}-81 \\
4976 \\
4895\end{array}$ \\
\hline I & $\begin{array}{r}2 s^{2} 2 p^{2}{ }^{3} P-1 D \\
1 D=1 S \\
3 P-1 S \\
{ }^{3} P-1 S\end{array}$ & $\begin{array}{l}19,145 \\
37,424 \\
56,569 \\
\end{array}$ & $\begin{array}{l}20,066 \\
22,913 \\
42,979 \\
\end{array}$ & $\begin{array}{r}921 \\
-14,511 \\
-13,590 \\
\end{array}$ & $\begin{array}{l}14,452 \\
29,977 \\
44,429 \\
\end{array}$ & $\begin{array}{l}15,227 \\
17,371 \\
32,598 \\
\end{array}$ & $\begin{array}{r}775 \\
-12,606 \\
-11,831 \\
\end{array}$ & $\begin{array}{r}9,347 \\
21,562 \\
30,809 \\
\end{array}$ & $\begin{array}{l}10,164 \\
11,455 \\
21,619 \\
\end{array}$ & $\begin{array}{r}817 \\
-10,107 \\
-9190 \\
\end{array}$ \\
\hline II & $\begin{array}{r}2 s 2 p^{3} S-3 S \\
5 S-3 D \\
5 S-3 P \\
5 S-3 P \\
3 D-1 D \\
3 D-1 D \\
3 P-1 P \\
\end{array}$ & $\begin{array}{r}137,640 \\
59,590 \\
80,933 \\
21,343 \\
68,820 \\
68,820 \\
\end{array}$ & $\begin{array}{r}137,192 \\
60,144 \\
82,492 \\
22,348 \\
67,011 \\
68,072 \\
\end{array}$ & $\begin{array}{r}-448 \\
554 \\
1559 \\
1005 \\
-1809 \\
-748 \\
\end{array}$ & $\begin{array}{r}109,992 \\
45,388 \\
61,708 \\
16,320 \\
54,996 \\
54,996 \\
\end{array}$ & $\begin{array}{r}108,462 \\
45,576 \\
62,552 \\
17,076 \\
51,945 \\
57,546 \\
\end{array}$ & $\begin{array}{r}-1530 \\
188 \\
844 \\
756 \\
-3051 \\
2550 \\
\end{array}$ & 8,224 & 11,167 & 2943 \\
\hline I & $\begin{array}{r}2 s 2 p^{3} S-3 S \\
5 S-3 D \\
5 S-3 P \\
5 D-3 P \\
3 D-1 D \\
3 D-1 P \\
3 P-3 \\
\end{array}$ & $\begin{array}{r}145,692 \\
62,728 \\
92,129 \\
29,401 \\
72,846 \\
72,846 \\
\end{array}$ & $\begin{array}{r}137,192 \\
60,144 \\
82,492 \\
22,348 \\
67,011 \\
68,072 \\
\end{array}$ & $\begin{array}{l}-8500 \\
-2584 \\
-9637 \\
-7053 \\
-5835 \\
-4774\end{array}$ & $\begin{array}{r}118,768 \\
48,869 \\
72,524 \\
23,655 \\
59,384 \\
59,384\end{array}$ & $\begin{array}{r}108,462 \\
45,576 \\
62,552 \\
16,976 \\
51,945 \\
57,546\end{array}$ & $\begin{array}{r}-10,306 \\
-3293 \\
-9972 \\
-6679 \\
-7439 \\
-1838\end{array}$ & & & \\
\hline II & $\begin{array}{r}2 p^{4}{ }^{3} P=1 D \\
1 D=1 S \\
3 P=1 S\end{array}$ & $\begin{array}{l}13,570 \\
45,369 \\
58,939\end{array}$ & $\begin{array}{l}14,426 \\
45,013 \\
59,439\end{array}$ & $\begin{array}{r}856 \\
-356 \\
500\end{array}$ & & & & & & \\
\hline I & $\begin{array}{r}2 p^{4}{ }^{3} P=1 D \\
1 D=1 S \\
3 P=1 S\end{array}$ & $\begin{array}{l}15,928 \\
50,778 \\
66,706\end{array}$ & $\begin{array}{l}14,426 \\
45,013 \\
59,039\end{array}$ & $\begin{array}{l}-1502 \\
-5765 \\
-6767\end{array}$ & & & & & & \\
\hline & $\begin{array}{r}\left.2 s^{2}\right) 2 p^{4} 3 P=1 D \\
1 D=1 S \\
3 P=1 S \\
3\end{array}$ & $\begin{array}{l}15,688 \\
18,040 \\
33,728\end{array}$ & $\begin{array}{l}15,791 \\
17,925 \\
33,716\end{array}$ & $\begin{array}{r}103 \\
-115 \\
-12\end{array}$ & & & & & & \\
\hline & $\begin{array}{r}\left.2 s^{2}\right) 2 p^{4}{ }^{3} P=1 D \\
1 D=1 S \\
3 P=1 S\end{array}$ & $\begin{array}{l}17,877 \\
20,476 \\
38,353\end{array}$ & $\begin{array}{l}15,791 \\
17,925 \\
33,176\end{array}$ & $\begin{array}{l}-2086 \\
-4551 \\
-4637\end{array}$ & & & & & & \\
\hline
\end{tabular}

II denotes highest approximation; I next highest.

higher orders are apparently more important for the latter, or put another way, the ${ }^{1} S$ is probably perturbed by adjacent levels. It is also interesting to notice that there are certain ratios of the separations holding for the lower approximation which still hold for the higher approximation. For example in $2 s 2 p^{3},\left({ }^{5} S-{ }^{3} S\right)$ is twice as large as either $\left({ }^{3} D-{ }^{1} D\right)$ or $\left({ }^{3} P-{ }^{1} P\right)$ in the lower approximation. ${ }^{9}$ The same relation holds in the higher approximation.

${ }^{\circ}$ This same ratio is obtained (See M. H. Johnson, Phys. Rev. 39, 209 (1932)) from the Slater-Condon relations.
The multiplet differences for the configuration, $2 s^{2} 2 p^{3}$, of $\mathrm{O}$ and $\mathrm{N}$ and for $2 s 2 p^{4}$ and $2 s^{2} 2 p^{4}$ for $\mathrm{O}$ are presented in Table XVIII. For the five electron configurations there are three approximations, III being the highest. Again $\left({ }^{4} S-{ }^{2} D\right)$ of $2 s^{2} 2 p^{3}$ is good in the lowest approximation but the other separations of that configuration are not. Agreement closer than 200 or $300 \mathrm{~cm}^{-1}$ must be regarded as fortuitous and it is doubtful

One cannot conclude from the apparent validity of these ratios in this and similar configurations, that first order calculations should be expected to hold. 
TABLE XVIII. Five and six electron configurations. Inter-multiplet separations.

\begin{tabular}{|c|c|c|c|c|c|c|c|c|}
\hline Element & Configuration and States & Obs. & Calc. III & $\begin{array}{l}\text { Obs.- } \\
\text { Calc. III }\end{array}$ & Calc. II & $\begin{array}{l}\text { Obs.- } \\
\text { Calc. II }\end{array}$ & Calc. I & $\begin{array}{l}\text { Obs.- } \\
\text { Calc. I }\end{array}$ \\
\hline $\mathrm{O}$ & $\begin{array}{r}2 s^{2} 2 p^{3} 4 S-2 D \\
-\quad 2 D=-2 P \\
-\quad 4 S^{5}-2 P \\
\end{array}$ & $\begin{array}{l}26,816 \\
13,651 \\
40,467 \\
\end{array}$ & $\begin{array}{l}27,102 \\
14,209 \\
41,311 \\
\end{array}$ & $\begin{array}{l}-286 \\
-558 \\
-844 \\
\end{array}$ & $\begin{array}{l}26,891 \\
12,418 \\
39,309 \\
\end{array}$ & $\begin{array}{r}-75 \\
1233 \\
1158 \\
\end{array}$ & $\begin{array}{l}27,000 \\
20,167 \\
47,167 \\
\end{array}$ & $\begin{array}{l}-184 \\
-6516 \\
-6700 \\
\end{array}$ \\
\hline $\mathrm{N}$ & $\begin{array}{r}2 s^{2} 2 p^{3}{ }^{4} S-2 D \\
2 D-2 P \\
{ }^{4} S-2 P \\
\end{array}$ & $\begin{array}{r}19,223 \\
9,617 \\
28,840 \\
\end{array}$ & $\begin{array}{l}19,054 \\
12,026 \\
31,080 \\
\end{array}$ & $\begin{array}{r}169 \\
-2409 \\
-2240 \\
\end{array}$ & $\begin{array}{r}19,403 \\
9,790 \\
29,193 \\
\end{array}$ & $\begin{array}{l}-180 \\
-173 \\
-353 \\
\end{array}$ & $\begin{array}{l}19,540 \\
16,111 \\
35,651\end{array}$ & $\begin{array}{l}-317 \\
-6494 \\
-6811\end{array}$ \\
\hline $\mathrm{O}$ & $\begin{array}{r}2 s 2 p^{4}{ }^{4} P-2 P \\
4 P-2 D \\
2 D-2 S \\
{ }^{4} P-2 S \\
\end{array}$ & $\begin{array}{l}92,719 \\
46,061 \\
29,719 \\
75,780 \\
\end{array}$ & $\begin{array}{l}93,808 \\
46,913 \\
30,060 \\
76,973 \\
\end{array}$ & $\begin{array}{r}-1089 \\
-852 \\
-341 \\
-1193 \\
\end{array}$ & $\begin{array}{l}94,935 \\
47,218 \\
28,908 \\
75,793 \\
\end{array}$ & $\begin{array}{r}-2216 \\
-1157 \\
811 \\
-18 \\
\end{array}$ & $\begin{array}{r}102,103 \\
50,154 \\
37,618 \\
87,772 \\
\end{array}$ & $\begin{array}{r}-9384 \\
-4093 \\
-7899 \\
-11,992\end{array}$ \\
\hline \multirow[t]{2}{*}{$\mathrm{O}$} & \multirow[t]{2}{*}{ 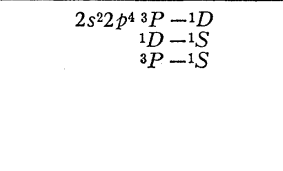 } & $\begin{array}{l}15,791 \\
17,925 \\
33,716\end{array}$ & $\begin{array}{l}15,907 \\
17,592 \\
33,499\end{array}$ & $\begin{array}{r}-116 \\
333 \\
217\end{array}$ & $\begin{array}{l}15,973 \\
16,261 \\
32,234\end{array}$ & $\begin{array}{r}-182 \\
1664 \\
1482\end{array}$ & $\begin{array}{l}16,516 \\
25,098 \\
41,614\end{array}$ & $\begin{array}{l}-725 \\
-7173 \\
-7898\end{array}$ \\
\hline & & \multicolumn{7}{|c|}{$\begin{array}{lrrr}\text { Calc. IV }\left({ }^{3} P-1 D\right) & 15,568 ; & (1 D-1 S) & 17,002 ; \\
\text { Obs.-Calc. } & 223 ; & 923 ; & 1146)\end{array}$} \\
\hline
\end{tabular}

For $s^{2} p^{3}$ and $s p^{4}$, III denotes the highest approximation, II next highest, I lowest.

For $s^{2} p^{4}$ there are four approximations: IV highest, III next highest, etc.

whether the greater error in the highest approximation for ${ }^{4} S-{ }^{2} D$ of $2 s^{2} 2 p^{3}$ has any significance. For $2 s^{2} 2 p^{4}$ the greater error in the highest approximation indicates that it is better to consider only the $2 p^{4}$ outside the closed $2 s^{2}$ shell. There are four approximations available for $2 s^{2} 2 p^{4}$ and $\left({ }^{3} P-{ }^{1} D\right)$ is not bad even in the lowest.

\section{Appendix I}

The relations developed here will now be examined by using the methods of the perturbation theory. For three electrons with coordinates $x_{1}, x_{2}$ and $x_{3}$, the Hamiltonian has the following form:

$$
H\left(x_{1} x_{2} x_{3}\right)=H_{0}+v\left(x_{1} x_{2} x_{3}\right)=H_{0}+v\left(x_{1} x_{2}\right)+v\left(x_{1} x_{3}\right)+v\left(x_{2} x_{3}\right)
$$

where $H_{0}$ includes the central field due to the nucleus and $v\left(x_{1} x_{2} x_{3}\right)$ is the interaction of the three electrons.

Let us consider the three electron state which is characterized by the quantum numbers $A, B$ and $C$ for the three electrons. Let $\Psi(A B C)$ represent the normalized wave function of this state including the first order terms. We can expand this $\Psi(A B C)$ in terms of the infinite set of one electron wave functions which are themselves properly normalized.

$$
\Psi(A B C)=\frac{1}{\sqrt{ } 6}\left|\begin{array}{lll}
A\left(x_{1}\right) & B\left(x_{1}\right) & C\left(x_{1}\right) \\
A\left(x_{2}\right) & B\left(x_{2}\right) & C\left(x_{2}\right) \\
A\left(x_{3}\right) & B\left(x_{3}\right) & C\left(x_{3}\right)
\end{array}\right|+\underset{a, b, c}{\Sigma^{\prime}} \kappa(a b c)-\frac{1}{\sqrt{ } 6}\left|\begin{array}{lll}
a\left(x_{1}\right) & b\left(x_{1}\right) & c\left(x_{1}\right) \\
a\left(x_{2}\right) & b\left(x_{2}\right) & c\left(x_{2}\right) \\
a\left(x_{3}\right) & b\left(x_{3}\right) & c\left(x_{3}\right)
\end{array}\right|,
$$

where

$$
\kappa(a b c)=\frac{V(A B C / a b c)}{E_{0}(A B C)-E_{0}(a b c)}
$$

and $a\left(x_{1}\right)$ is the wave function of the state $a$ of the one electron problem which has the same central field and the prime on the sum indicates that the term $A B C$ is omitted. $E_{0}(A B C)$ is the energy of the state $A B C$ of the three electron problem if the interaction of the electrons is completely neglected.

$$
E_{0}(A B C)=E(A)+E(B)+E(C) .
$$

The change in the energy of the state $A B C$ due to the elect;ostatic interaction, including terms of the first and second order, can be written

$$
E(A B C)=\int \Psi(A B C) v\left(x_{1} x_{2} x_{3}\right) \Psi^{*}(A B C) d x_{1} d x_{2} d x_{3}
$$

There will be no terms in $E(A B C)$ in which the quantum numbers of the antisymmetric combination of one electron wave functions will differ for more than two electrons. On this basis the energy $E(A B C)$ may be written in three parts,

$$
E(A B C)=E_{1}(A B C)+E_{2}(A B C)+E_{3}(A B C)
$$

where $E_{1}(A B C), E_{2}(A B C)$ and $E_{3}(A B C)$ contain only terms which differ in zero, one and two electrons, respectively. 
For $E_{1}(A B C), E_{2}(A B C)$ and $E_{3}(A B C)$ in terms of the expanded wave functions, we find

$$
\begin{aligned}
& E_{1}(A B C)=V(A B C / A B C) \\
& E_{2}(A B C)=\Sigma\left\{\kappa^{*}(A B n) V(A B C / A B n)+\kappa(A B n) V(A B n / A B C)+\kappa^{*}(A n C) V(A B C / A n C)+\kappa(A n C) V(A n C / A B C)\right. \\
& \left.n \quad+\kappa^{*}(n B C) V(A B C / n B C)+\kappa(n B C) V(n B C / A B C)\right\} \\
& =\Sigma\{2 \kappa(A B n) V(A B C / A B n)+2 \kappa(A n C) V(A B C / A n C)+2 \kappa(n B C) V(A B C / n B C)\}, \\
& E_{3}(A B C)=\sum_{m, n}\{2 \kappa(A m n) V(A B C / A m n)+2 \kappa(m n C) V(A B C / m n C)+2 \kappa(m B n) V(A B C / m B n)\}, \\
& \text { where } \quad V(A B C / A m n)=1 / 6 \int\left|\begin{array}{lll}
A\left(x_{1}\right) & B\left(x_{1}\right) & C\left(x_{1}\right) \\
A\left(x_{2}\right) & B\left(x_{2}\right) & C\left(x_{2}\right) \\
A\left(x_{3}\right) & B\left(x_{3}\right) & C\left(x_{3}\right)
\end{array}\right| v\left(x_{1} x_{2} x_{3}\right)\left|\begin{array}{lll}
A\left(x_{1}\right) & m\left(x_{1}\right) & n\left(x_{1}\right) \\
A\left(x_{2}\right) & m\left(x_{2}\right) & n\left(x_{2}\right) \\
A\left(x_{3}\right) & m\left(x_{3}\right) & n\left(x_{3}\right)
\end{array}\right| d x_{1} d x_{2} d x_{3} .
\end{aligned}
$$

Since we wish to examine certain relations between the energies of the three electron problem and those of the two electron problem we must now study the latter. The central field will be taken as the same as that for the three electron problem so that if the interaction of the electrons is neglected, the energy states for a single electron are the same for both cases. The change in energy of the state $A B$ due to the electrostatic interaction may again be written in three parts.

$$
E(A B)=E_{1}(A B)+E_{2}(A B)+E_{3}(A B),
$$

where $E_{1}(A B), E_{2}(A B)$ and $E_{3}(A B)$ contain only terms which differ in zero, one and two electrons, respectively. In terms of the expanded wave functions of the two electron problem (the $\lambda$ 's are the expansion coefficients), we find

$$
\begin{aligned}
& E_{1}(A B)=V(A B / A B), \\
& E_{2}(A B)=\sum_{n}\{2 \lambda(A B / A n) V(A B / A n)+2 \lambda(A B / n B) V(A B / n B)\}, \\
& E_{3}(A B)=\sum_{m, n}\{2 \lambda(A B / m n) V(A B / m n)\}, \\
& \text { where } \quad \begin{aligned}
A(A B / m n)=1 / 2 \int\left|\begin{array}{ll}
A\left(x_{1}\right) & B\left(x_{1}\right) \\
A\left(x_{2}\right) & B\left(x_{2}\right)
\end{array}\right| v\left(x_{1} x_{2}\right)\left|\begin{array}{ll}
m\left(x_{1}\right) & n\left(x_{1}\right) \\
m\left(x_{2}\right) & n\left(x_{2}\right)
\end{array}\right| d x_{1} d x_{2}
\end{aligned} \\
& \text { and } \lambda(A B / m n)=\frac{V(A B / m n)}{E_{0}(A B)-E_{0}(m n)} .
\end{aligned}
$$

We find that the $V$ 's of the three electron problem can be written in terms of those of two electrons due largely to Eq. (44).

$$
\begin{aligned}
v\left(x_{1} x_{2} x_{3}\right) & =v\left(x_{1} x_{2}\right)+v\left(x_{1} x_{3}\right)+v\left(x_{2} x_{3}\right), \\
V(A B C / A B C) & =V(B C / B C)+V(A C / A C)+V(A B / A B), \\
V(A B C / A B n) & =V(B C / B n)+V(A C / A n), \\
V(A B C / A m n) & =V(B C / m n) .
\end{aligned}
$$

Using the above relations between the V's we can find the $\kappa$ 's in terms of the $\lambda$ 's.

$$
\begin{aligned}
\kappa(A m n) & =\lambda(B C / m n), \\
\kappa(A B n) & =\lambda(B C / B n)+\lambda(A C / A n) .
\end{aligned}
$$

These relations enable us to write the electrostatic energies of the three electron problem in terms of those of the two electron problem.

$$
E_{1}(A B C)=V(A B / A B)+V(A C / A C)+V(B C / B C)=E_{1}(A B)+E_{1}(A C)+E_{1}(B C) .
$$

This is a relation between the first order terms of the electrostatic interaction. If we include the energy in the central field we find

$$
W^{1}(A B C)=W^{1}(A B)+W^{1}(A C)+W^{1}(B C)-W(A)-W(B)-W(C) .
$$

Since this is the same as Eq. (4), this shows that (4) is exact to the first order terms. For $E_{2}(A B C)$ and $E_{3}(A B C)$ we find

$$
\begin{aligned}
E_{2}(A B C)= & \sum_{n}\{2[\lambda(B C / B n)+\lambda(A C / A n)][V(B C / B n)+V(A C / A n)]+2[\lambda(A B / A n)+\lambda(B C / n C)] \\
& \quad \times[V(A B / A n)+V(B C / n C)]+2[\lambda(A B / n B)+\lambda(A C / n C)][V(A B / n B)+V(A C / n C)]\}, \\
E_{2}(A B C)= & E_{2}(A B)+E_{2}(A C)+E_{2}(B C)+\text { cross terms in }(52), \\
E_{3}(A B C)=\sum_{m, n}\{2 \lambda(B C / m n) V(B C / m n)+2 \lambda(A B / m n) V(A B / m n)+2 \lambda(A C / m n) V(A C / m n)\}, & .
\end{aligned}
$$

If it were not for the cross terms in (52) we would again get Eq. (4) when (53) and (54) are added to (51). Except for the cross terms in (52), Eq. (4) would hold up to the second order. We conclude that Eq. (4) which relates the energies 
of the three, two and one electron problems, is exact up to the first order and holds also for parts of the second order.

Let us now consider four electrons in the same central field. The Hamiltonian of the problem will have the form

$$
H\left(x_{1} x_{2} x_{3} x_{4}\right)=H_{0}\left(x_{1} x_{2} x_{3} x_{4}\right)+\sum_{j>i=1}^{4} v\left(x_{i} x_{j}\right)
$$

where the central field is included in $H_{0}$. The wave function (including first order terms) of the state which is characterized by the quantum numbers $A, B, C$ and $D$ for the four electrons may be written

$$
\Psi(A B C D)=\frac{1}{\sqrt{24}}\left|\begin{array}{llll}
A\left(x_{1}\right) & B\left(x_{1}\right) & C\left(x_{1}\right) & D\left(x_{1}\right) \\
A\left(x_{2}\right) & B\left(x_{2}\right) & C\left(x_{2}\right) & D\left(x_{2}\right) \\
A\left(x_{3}\right) & B\left(x_{3}\right) & C\left(x_{3}\right) & D\left(x_{3}\right) \\
A\left(x_{4}\right) & B\left(x_{4}\right) & C\left(x_{4}\right) & D\left(x_{4}\right)
\end{array}\right|+\Sigma_{m n o p}^{\prime} \mu(m n o p) \frac{1}{\sqrt{24}}\left|\begin{array}{llll}
m\left(x_{1}\right) & n\left(x_{1}\right) & o\left(x_{1}\right) & p\left(x_{1}\right) \\
m\left(x_{2}\right) & n\left(x_{2}\right) & o\left(x_{2}\right) & p\left(x_{2}\right) \\
m\left(x_{3}\right) & n\left(x_{3}\right) & o\left(x_{3}\right) & p\left(x_{3}\right) \\
m\left(x_{4}\right) & n\left(x_{4}\right) & o\left(x_{4}\right) & p\left(x_{4}\right)
\end{array}\right| .
$$

where $\mu(m n o p)=\frac{V(A B C D / m n o p)}{E_{0}(A B C D)-E_{0}(m n o p)}=0$ if mnop differs from $A B C D$ in the quantum numbers of more than two electrons.

The change in the energy $E(A B C D)$ due to the electrostatic interaction, including second order terms, may be split into three parts as before.

$$
E(A B C D)=E_{1}(A B C D)+E_{2}(A B C D)+E_{3}(A B C D) .
$$

For $E_{1}(A B C D), E_{2}(A B C D)$ and $E_{3}(A B C D)$ we find

$$
\begin{aligned}
E_{1}(A B C D)= & V(A B / A B)+V(A C / A C)+V(A D / A D)+V(B C / B C)+V(B D / B D)+V(C D / C D), \\
E_{1}(A B C D)= & E_{1}(A B C)+E_{1}(A B D)+E_{1}(A C D)+E_{1}(B C D)-E_{1}(A B)-E_{1}(A C)-E_{1}(A D)-E_{1}(B C)-E_{1}(B D)-E_{1}(C D), \quad(58) \\
E_{2}(A B C D)= & \sum_{n}\{2 \mu(A B C n)[V(A D / A n)+V(B D / B n)+V(C D / C n)] \\
& +2 \mu(A B n D)[V(A C / A n)+V(B C / B n)+V(C D / n D)]+2 \mu(A n C D)[V(A B / A n)+V(B C / n C)+V(B D / n D)] \\
& \quad+2 \mu(n B C D)[V(A B / n B)+V(A C / n C)+V(A D / n D)]\}, \\
E_{2}(A B C D)= & E_{2}(A B C)+E_{2}(A B D)+E_{2}(A C D)+E_{2}(B C D)-E_{2}(A B)-E_{2}(A C)-E_{2}(A D)-E_{2}(B C)-E_{2}(B D)-E_{2}(C D), \quad(59) \\
E_{3}(A B C D)= & \sum_{m, n}\{2 \mu(A B m n) V(C D / m n)+2 \mu(A m C n) V(B D / m n)+2 \mu(m B C n) V(A D / m n) \\
& \quad+2 \mu(m B n D) V(A C / m n)+2 \mu(A m n D) V(B C / m n)+2 \mu(m n C D) V(A B / m n)\}, \\
E_{3}(A B C D)= & E_{3}(A B C)+E_{3}(A B D)+E_{3}(A C D)+E_{3}(B C D)-E_{3}(A B)-E_{3}(A C)-E_{3}(A D)-E_{3}(B C)-E_{3}(B D)-E_{3}(C D) .
\end{aligned}
$$

If we include the energy of the unperturbed problem we find for the total energy in second order.

$W^{2}(A B C D)=W^{2}(A B C)+W^{2}(A B D)+W^{2}(A C D)+W^{2}(B C D)-W^{2}(A B)-W^{2}(A C)-W^{2}(A D)-W^{2}(B C)$

$$
-W^{2}(B D)-W^{2}(C D)+W(A)+W(B)+W(C)+W(D) \text {. }
$$

This relation is the same as Eq. (7) so we conclude that (7) is exact up to and including the second order. It is very probable that (7) holds for parts of the third and higher orders. Up to the second order one can verify the relations for more than four electrons in the same way, although the equations become somewhat lengthy. Inclusion of the third and higher orders, however, greatly increases the complication.

\section{Appendix II}

In the configuration $p^{2}$ consider the two states with $M_{S}=0, M_{L}=1$. We know that these two states together give ${ }^{3} P$ and ${ }^{1} D$, each being some linear combination of the two. In order to find this linear combination, let us consider in Table IV for $p^{3}$ the two states which are ${ }^{2} D$ alone. These two states must have the same pair energy. If we write the pair energy of two electrons as $w\left(m_{s} m_{l} m_{s}{ }^{\prime} m_{l}{ }^{\prime}\right)$ and the total pair energy for ${ }^{2} D$ as $w\left({ }^{2} D\right)$ we have the following relations

$$
\begin{gathered}
w\left({ }^{2} D\right)=w(1 / 21,1 / 20)+w(1 / 21,-1 / 21)+w(1 / 20,-1 / 21), \\
{ }^{3} P \quad \begin{array}{c}
1 D \\
w\left({ }^{2} D\right)
\end{array}=w(1 / 21,-1 / 21)+w(1 / 21,-1 / 20)+w(-1 / 21,-1 / 20) . \\
{ }^{3} D
\end{gathered}
$$

In (62), the first pair energy is that of ${ }^{3} P$ of $p^{2}$ (compare Table II) and the second that of ${ }^{1} D$. In (63), the first is ${ }^{1} D$ and the last ${ }^{3} P$. Since the pair energies must be equal in both cases we have (64)

$$
w(1 / 20,-1 / 21)=w(1 / 21,-1 / 20) \text {. }
$$

If we go back now to the two states of $p^{2}$ having $M_{S}=0$ and $M_{L}=1$, we see that (64) shows they have the same pair energy and hence the two states must have the same linear combination of ${ }^{3} P$ and ${ }^{1} D$. The two states together give ${ }^{3} P+{ }^{1} D$ so each state is $1 / 2{ }^{3} P+1 / 2{ }^{1} D$. 
Let us consider the three states with $M_{S}=0$ and $M_{L}=0$ from the configuration $p^{2}$. We know that these three states together give ${ }^{3} P,{ }^{1} D$ and ${ }^{1} S$. If we go to Table VIII for configuration $p^{4}$ there are four states which are uniquely $p^{4}{ }^{3} P$. Since these must have the same pair energy Eqs. (65), (66) and (67) result.

$$
\begin{aligned}
w\left(p^{4} P\right)=w(1 / 21,1 / 20)+w(1 / 21,1 / 2-1)+w(1 / 21,-1 / 21)+w(1 / 20,1 / 2-1)+w(1 / 20,-1 / 21) & +w(1 / 2-1,-1 / 21), \\
w\left(p^{4} 3\right)=w(1 / 21,1 / 20)+w(1 / 21,1 / 2-1)+w(1 / 21,-1 / 20)+w(1 / 20,1 / 2-1)+w(1 / 20,-1 / 20) & \\
& +w(1 / 2-1,-1 / 20), \quad(66) \\
w\left(p^{4} 3\right)=w(1 / 21,1 / 20)+w(1 / 21,1 / 2-1)+w(1 / 21,-1 / 2-1)+w(1 / 201 / 2-1)+w(1 / 20,-1 / 2-1) & +w(1 / 2-1,-1 / 2-1) .
\end{aligned}
$$

Utilizing knowledge of the pair energies of other states of $p^{2}$, and noting that if the signs of all the quantum numbers in a pair are changed the pair energy is unchanged, we get the following relations:

$$
\begin{aligned}
w(1 / 20,-1 / 20) & =1 / 2 w\left({ }^{1} D\right)-1 / 2 w\left({ }^{3} P\right)+w(1 / 21,-1 / 2-1), \\
w(1 / 2-1,-1 / 21) & =w(1 / 21,-1 / 2-1) .
\end{aligned}
$$

In addition the three states together give ${ }^{3} P,{ }^{1} D$ and ${ }^{1} S$.

$$
w(1 / 20,-1 / 20)+2 w(1 / 21,-1 / 2-1)=w\left({ }^{3} P\right)+w\left({ }^{1} D\right)+w\left({ }^{1} S\right)
$$

Hence we have (71) and (72)

$$
\begin{aligned}
w(1 / 21,-1 / 2-1) & =1 / 2 w\left({ }^{3} P\right)+1 / 6 w\left({ }^{1} D\right)+1 / 3 w\left({ }^{1} S\right), \\
w(1 / 20,-1 / 20) & =2 / 3 w\left({ }^{1} D\right)+1 / 3 w\left({ }^{1} S\right) .
\end{aligned}
$$

This means that in the set $M_{S}=0, M_{L}=0$ the first and third states are $1 / 2{ }^{3} P+1 / 6{ }^{1} D+1 / 3{ }^{1} S$ and the second is $2 / 3{ }^{1} D+1 / 3{ }^{1} S$.

Eqs. (64) and (69) indicate that the division into multiplets is the same for two states which differ either in the signs of all the $m_{s}$ or all the $m_{l}$. The state which differs from another state in the signs of all the $m_{s}$ and all the $m_{l}$ is simply the corresponding state from the half of the Pauli table with $M_{S}+M_{L}<0$ and also has the same division into multiplets.

\section{$p^{3}$}

From the configuration $p^{3}$ there are multiplets ${ }^{4} S,{ }^{2} D$ and ${ }^{2} P$. In the set with $M_{S}=1 / 2$ and $M_{L}=1$ (see Table IV), we find that each of the states has the same pair energy and hence each state is given by $1 / 2^{2} D+1 / 2{ }^{2} P$ since the two together give ${ }^{2} P+{ }^{2} D$. The same is true for the set with $M_{S}=-1 / 2$, and $M_{L}=1$.

In the set with $M_{S}=1 / 2$ and $M_{L}=0$ there are three states which together give ${ }^{4} S,{ }^{2} D$ and ${ }^{2} P$. Since we know that the pair energies of a state $(A B C)$ must be the same as for $(A B)+(A C)+(B C)$, we have

$$
w(A B C)=w(A B)+w(A C)+w(B C)
$$

where $w(A B C)$ is used here to represent the pair energies of $(A B C)$. Utilizing (73), we find from the states which are already known in Table IV the following relations

$$
\begin{aligned}
w\left(p^{3}{ }^{4} S\right) & =3 w\left(p^{2}{ }^{3} P\right), \\
w\left(p^{3}{ }^{2} D\right) & =3 / 2 w\left(p^{3} P\right)+3 / 2 w\left(p^{2}{ }^{1} D\right), \\
w\left(p^{3}{ }^{2} P\right) & =3 / 2 w\left(p^{2}{ }^{3} P\right)+5 / 6 w\left(p^{2}{ }^{1} D\right)+2 / 3 w\left(p^{2}{ }^{1} S\right) .
\end{aligned}
$$

From the three states with $M_{S}=1 / 2$ and $M_{L}=0$ we have

$$
\begin{aligned}
& w(1 / 21,1 / 20,-1 / 2-1)=2 w\left(p^{2}{ }^{3} P\right)+2 / 3 w\left(p^{2} 1 D\right)+1 / 3 w\left(p^{2}{ }^{1} S\right), \\
& w(1 / 21,1 / 2-1,-1 / 20)=2 w\left(p^{2}{ }^{3} P\right)+w\left(p^{2}{ }^{1} D\right), \\
& w(1 / 20,1 / 2-1,-1 / 21)=2 w\left(p^{2}{ }^{3} P\right)+2 / 3 w\left(p^{2} D\right)+1 / 3 w\left(p^{2}{ }^{1} S\right) .
\end{aligned}
$$

We wish to find $w(1 / 21,1 / 20,-1 / 2-1), w(1 / 21,1 / 2-1,-1 / 20)$ and $w(1 / 20,1 / 2-1,-1 / 21)$ in terms of $w\left(p^{34} S\right)$, $w\left(p^{3} D\right)$ and $w\left(p^{3} P\right)$ so that all the Eqs. (74) to (79) are satisfied simultaneously. If we solve (74), (75) and (76) for $w\left(p^{2}{ }^{3} P\right), w\left(p^{2} D\right)$ and $w\left(p^{2}{ }^{1} S\right)$ and substitute these values in (77), (78) and (79), we get the following

$$
\begin{aligned}
& w(1 / 21,1 / 20,-1 / 2-1)=1 / 3 w\left(p^{3}{ }^{4} S\right)+1 / 6 w\left(p^{3}{ }^{2} D\right)+1 / 2 w\left(p^{3}{ }^{2} P\right), \\
& w(1 / 21,1 / 2-1,-1 / 20)=1 / 3 w\left(p^{3} S\right)+2 / 3 w\left(p^{3} 2\right), \\
& w(1 / 20,1 / 2-1,-1 / 21)=1 / 3 w\left(p^{3} S\right)+1 / 6 w\left(p^{3} 2\right)+1 / 2 w\left(p^{3}{ }^{2} P\right) .
\end{aligned}
$$

The relations (80), (81) and (82) for the pair energies give the proper linear combinations found in Table IV for the three states of the set $M_{S}=1 / 2, M_{L}=0$.

It may seem that this is somewhat involved, but it is nearly always possible, with a little practice, to determine the coefficients immediately from inspection. For example, in the last case it is apparent since $p^{3}{ }^{2} P$ occurs (see Table IV, last column) only with $p^{2}{ }^{1} S$, that it will occur in the first and third states of the set with coefficient $1 / 2$. The second state 
has a ${ }^{1} D$ in the multiplets from pairs but no ${ }^{2} P$ in the $p^{3}$ multiplets, so ${ }^{2} D$ must occur with the coefficient $2 / 3$. The first and third states must have the same linear combination and the sum of the ${ }^{2} D$ coefficients is unity so ${ }^{2} D$ occurs in the first and third states with coefficient $1 / 6$. Since for each state the sum of the coefficients must be unity, the coefficient of ${ }^{4} S$ is $1 / 3$ for each state.

It is not necessary to know the exact linear combination of all the states of Table IV in order to find the energies of the various multiplets but the information is useful in finding the linear combinations in more complicated configurations.

\section{$s p^{2}$}

From the configuration $s p^{2}$ there are multiplets ${ }^{4} P,{ }^{2} P,{ }^{2} D$ and ${ }^{2} S$. Each of the states in Table $\mathrm{V}$ with $M_{S}=3 / 2$ is assigned to ${ }^{4} P$ alone and those with $M_{L}=2$ belong to ${ }^{2} D$. In the set with $M_{S}=1 / 2$ and $M_{L}=1$ three of the possible four states will be represented, ${ }^{4} P,{ }^{2} P$ and ${ }^{2} D$. Using (73) and the information available about ${ }^{4} P$ and ${ }^{2} D$ we find

$$
\begin{aligned}
& w\left(s p^{2}{ }^{4} P\right)=w\left(p^{2}{ }^{3} P\right)+2 w\left(s p^{3} P\right), \\
& w\left(s p^{2}{ }^{2} D\right)=w\left(p^{2}{ }^{1} D\right)+3 / 2 w\left(s p^{3} P\right)+1 / 2 w\left(s p^{1} P\right),
\end{aligned}
$$

Considering the whole set $M_{S}=1 / 2, M_{L}=1$ we have

$$
w\left(s p^{24} P\right)+w\left(s p^{2} D\right)+w\left(s p^{2} P\right)=2 w\left(p^{2}{ }^{3} P\right)+w\left(p^{2} 1 D\right)+4 w\left(s p^{3} P\right)+2 w\left(s p^{1} P\right) .
$$

With (83) and (84) this gives

$$
w\left(s p^{2}{ }^{2} P\right)=w\left(p^{2}{ }^{3} P\right)+1 / 2 w\left(s p^{3} P\right)+3 / 2 w\left(s p^{1} P\right) .
$$

From the individual states of the $M_{S}=1 / 2, M_{L}=1$ set, we have

$$
\begin{aligned}
w(1 / 2,1 / 21,-1 / 20) & =w(1 / 2,1 / 20,-1 / 21)=1 / 2 w\left(p^{2}{ }^{3} P\right)+1 / 2 w\left(p^{2} 1 D\right)+3 / 2 w\left(s p^{3} P\right)+1 / 2 w\left(s p^{1} P\right), \\
w(1 / 2,1 / 21,1 / 20) & =w\left(p^{2}{ }^{3} P\right)+w\left(s p^{3} P\right)+w\left(s p^{1} P\right) .
\end{aligned}
$$

If now we solve (83), (84) and (86) for $w\left(p^{2}{ }^{3} P\right), w\left(s p^{3} P\right)$ and $w\left(s p^{1} P\right)$ in terms of $w\left(s p^{2}{ }^{4} P\right)$, w(s $\left.p^{2} D\right), w\left(s p^{2}{ }^{2} P\right)$ and $w\left(p^{2} 1 D\right)$ and put them into (87) and (88) we find

$$
\begin{aligned}
w(1 / 2,1 / 21,-1 / 20) & =w(1 / 2,1 / 20,-1 / 21)=1 / 3 w\left(s p^{2}{ }^{4} P\right)+1 / 2 w\left(s p^{2}{ }^{2} D\right)+1 / 6 w\left(s p^{2}{ }^{2} P\right), \\
w(1 / 2,1 / 21,1 / 20) & =1 / 3 w\left(s p^{2}{ }^{4} P\right)+2 / 3 w\left(s p^{2}{ }^{2} P\right) .
\end{aligned}
$$

These relations for the pair energies give the linear combinations for the three states with $M_{S}=1 / 2$ and $M_{L}=1$ found in Table V.

In the set of states with $M_{S}=1 / 2$ and $M_{L}=0$ all four possible multiplets, ${ }^{4} P,{ }^{2} P,{ }^{2} D$, and ${ }^{2} S$, are represented. Considering the whole set together we have

$$
w\left(s p^{2}{ }^{4} P\right)+w\left(s p^{2}{ }^{2} P\right)+w\left(s p^{2}{ }^{2} D\right)+w\left(s p^{2}{ }^{2} S\right)=2 w\left(p^{2}{ }^{3} P\right)+w\left(p^{21} D\right)+w\left(p^{2}{ }^{1} S\right)+11 / 2 w\left(s p{ }^{3} P\right)+5 / 2 w\left(s p{ }^{1} P\right) .
$$

With the help of (83), (84) and (86) we find from (91)

$$
w\left(s p^{2}{ }^{2} S\right)=w\left(p^{2}{ }^{1} S\right)+3 / 2 w\left(s p^{3} P\right)+1 / 2 w\left(s p^{1} P\right)
$$

For the four states we get the following relations for the pair energies

$$
\begin{aligned}
w(1 / 2,1 / 21,-1 / 2-1) & =w(1 / 2,1 / 2-1,-1 / 21)=1 / 2 w\left(p^{2}{ }^{3} P\right)+1 / 6 w\left(p^{2} 1 D\right) \\
& +1 / 3 w\left(p^{2} 1 S\right)+3 / 2 w\left(s p^{3} P\right)+1 / 2 w\left(s p^{1} P\right), \\
w(1 / 2,1 / 20,-1 / 20) & =2 / 3 w\left(p^{2} 1 D\right)+1 / 3 w\left(p^{2}{ }^{1} S\right)+3 / 2 w\left(s p^{3} P\right)+1 / 2 w\left(s p^{1} P\right), \\
w(-1 / 2,1 / 21,1 / 2-1) & =w\left(p^{2} P\right)+w\left(s p^{3} P\right)+w\left(s p^{1} P\right) .
\end{aligned}
$$

Solving (83), (84), (86) and (92) for $w\left(p^{2}{ }^{3} P\right), w\left(p^{1} S\right), w\left(s p^{3} P\right)$ and $w\left(s p^{1} P\right)$ in terms of $w\left(s p^{2}{ }^{4} P\right), w\left(s p^{2}{ }^{2} P\right)$, $w\left(s p^{2}{ }^{2} D\right), w\left(s p^{2}{ }^{2} S\right)$ and $w\left(p^{2} 1 D\right)$ as above and putting them in (93), (94) and (95) we find

$$
\begin{aligned}
w(1 / 2,1 / 21,-1 / 2-1) & =w(1 / 2,1 / 2-1,-1 / 21)=1 / 3 w\left(s p^{2}{ }^{4} P\right)+1 / 6 w\left(s p^{2}{ }^{2} P\right)+1 / 6 w\left(s p^{2}{ }^{2} D\right)+1 / 3 w\left(s p^{2}{ }^{2} S\right), \\
w(1 / 2,1 / 20,-1 / 20) & =1 / 3 w\left(s p^{2}{ }^{2} S\right)+2 / 3 w\left(s p^{2}{ }^{2} D\right),
\end{aligned}
$$$$
w(-1 / 2,1 / 21,1 / 2-1)=1 / 3 w\left(s p^{2}{ }^{4} P\right)+2 / 3 w\left(s p^{2}{ }^{2} P\right) \text {. }
$$

The relations (96), (97) and (98) give the proper linear combinations found in Table V. For this configuration the inspection method gives the coefficients almost immediately.

No further particular examples are worked out here in detail as we believe the method is amply illustrated above. The coefficients can nearly always be found most easily by the inspection method for which a certain amount of familiarity with the Pauli tables is indeed helpful. There are numerous regularities to guide one which unfortunately cannot be pointed out here in detail. 